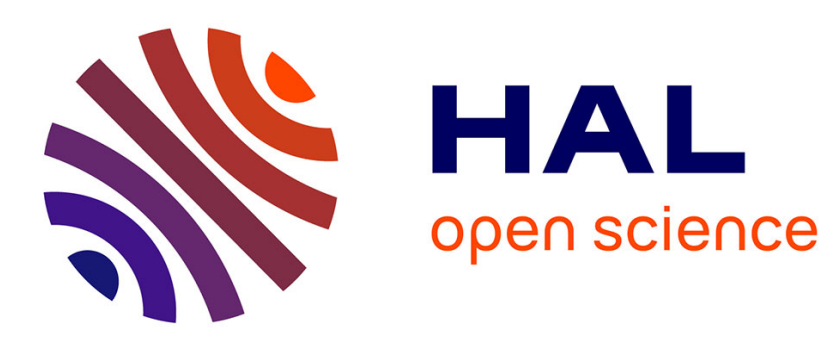

\title{
Algebraic totality, towards completeness
}

Christine Tasson

\section{To cite this version:}

Christine Tasson. Algebraic totality, towards completeness. Logic in Computer Science, Jul 2009, Brasilia, Brazil. pp.325-340, 10.1007/978-3-642-02273-9 . hal-00440750

\section{HAL Id: hal-00440750 \\ https://hal.science/hal-00440750}

Submitted on 11 Dec 2009

HAL is a multi-disciplinary open access archive for the deposit and dissemination of scientific research documents, whether they are published or not. The documents may come from teaching and research institutions in France or abroad, or from public or private research centers.
L'archive ouverte pluridisciplinaire HAL, est destinée au dépôt et à la diffusion de documents scientifiques de niveau recherche, publiés ou non, émanant des établissements d'enseignement et de recherche français ou étrangers, des laboratoires publics ou privés. 


\title{
Algebraic totality, towards completeness.
}

\author{
Christine Tasson \\ Preuves, Programmes, Systèmes
}

\begin{abstract}
Finiteness spaces constitute a categorical model of Linear Logic whose objects can be seen as linearly topologised spaces, (a class of topological vector spaces introduced by Lefschetz in 1942) and morphisms as continuous linear maps. First, we recall definitions of finiteness spaces and describe their basic properties deduced from the general theory of linearly topologised spaces. Then we give the interpretation of LL with an algebraic approach. Second, thanks to separation properties, we can introduce an algebraic notion of totality candidate in the framework of linearly topologised spaces: a totality candidate is a closed affine subspace which does not contain 0 . We show that finiteness spaces with totality candidates constitute a model of classical LL. Finally, we give a barycentric simply typed lambda-calculus, with booleans $\mathcal{B}$ and a conditional operator, which can be interpreted in this model. We prove completeness at type $\mathcal{B}^{n} \rightarrow \mathcal{B}$ for every $n$ by an algebraic method.
\end{abstract}

\section{Introduction}

In the 80's, Girard has been led to introduce linear logic (LL) after a denotational investigation of system F. The basic idea of LL is to decompose the intuitionistic implication into a linear one and an exponential modality. Many intuitions behind LL are rooted in linear algebra and relate algebraic concepts with operational ones. For instance, a linear function in the LL sense is a program (or a proof) which uses its argument exactly once and LL shows that this idea is similar to linearity in the algebraic sense. Can we use vector spaces and linear maps for interpreting LL? In the exponential-free fragment of LL, this is quite easy, since all vector spaces can stay finite dimensional: it is sufficient to take the standard relational interpretation of a formula (which is a set) and to build the vector space generated by this set. However, the exponential modality introduces infinite dimension and some topology is needed for controlling the size of dual spaces. Indeed, we want all spaces to be reflexive, that is, isomorphic to their second dual, because duality corresponds to linear negation which is involutive.

There are various ways for defining interpretations with linear spaces. Among them, the interpretations based on linearly topologised spaces $[2,6]$ have the feature of not requiring any topology on the field $\mathbb{k}$. This is quite natural, since the topology of the field is never used for interpreting proofs. Introduced first by Lefschetz in [14], these spaces are geometrically quite unintuitive (their basic opens are linear subsets whereas usual basic opens are balls). They provide nevertheless the simplest setting where formulæ of LL can be seen as (topological) linear spaces as shown by Ehrhard when he introduced finiteness spaces [6].

There are two ways of considering finiteness spaces:

Relational finiteness spaces: they can be seen as a refinement of the relational semantics 
of linear logic, in which the semantics of proofs is the same as the standard one (proofs are interpreted as relations).

Linear finiteness spaces: given a field, any relational finiteness space gives rise to a linearly topologised vector space. The category of linear finiteness spaces and continuous linear functions constitutes a model of linear logic. Besides, a linear finiteness space and its dual with the evaluation as pairing is a Chu space [3]. The proofs of linear logic are interpreted as multilinear hypocontinuous maps (hypocontinuity is between separate continuity and continuity). Our description of proofs (c.f. Annex B) is closed to that of Game Categories of Lafont and Streicher [13].

For describing these categories, we use the duality presentation whose importance has been emphasised by models of Classical Linear Logic such as phase semantics. Even the definition of coherence spaces [9], usually described by means of a binary symmetric and reflexive coherence relation $\Xi_{X}$ on a set $|X|$, can be reformulated through duality [1]. We will freely use the terminology of [11] — a survey of the different duality presentations and in particular of models of linear logic by double orthogonal. The partial orthogonality between the subsets $c$ and $c^{\prime}$ of $|X|$ is given by

$$
c \perp_{\text {coh }} c^{\prime} \Longleftrightarrow \sharp\left(c \cap c^{\prime}\right) \leq 1 \text {. }
$$

A coherence space can then be seen as a pair $X=(|X|, \mathcal{C}(X))$ where $|X|$ is a set and $\mathcal{C}(X)$ is a subset of $\mathcal{P}(|X|)$ (the powerset of $|X|)$, which is $\perp_{\text {coh-closed, that is }}$ equal to its second dual for the duality induced by the orthogonality: $\mathcal{C}(X)=\mathcal{C}(X)^{\perp \perp}$. The elements of $\mathcal{C}(X)$ are the cliques of $X$, those of $\mathcal{C}(X)^{\perp}$ are the anticliques. The category of coherence spaces and cliques is an orthogonality category.

The category of relational finiteness spaces and finitary relations (a refinement of standard relations) also constitute an orthogonality category with respect to the finite orthogonality defined as follows: let $u$ and $u^{\prime}$ be two sets,

$$
u \perp_{\text {fin }} u^{\prime} \Longleftrightarrow \sharp\left(u \cap u^{\prime}\right)<\infty .
$$

A relational finiteness space is a pair $X=(|X|, \mathcal{F}(X))$ where $|X|$ is a countable set and the set $\mathcal{F}(X)$ of finitary parts is $\perp_{\text {fin }}$-closed. The elements of $\mathcal{F}(X)^{\perp}$ are the antifinitary parts. We carry the relational finiteness in the linear world by considering the linear subspace of $\mathbb{k}^{|X|}$ generated by finitary linear combinations, that is families $x=\left(x_{a}\right)_{a \in|X|}$ such that their support $|x|$ is finitary. The finite orthogonality between supports $\left(|x| \perp_{\text {fin }}|x|^{\prime}\right)$ implies that the pairing between a finitary linear combination $x$ and antifinitary one $x^{\prime}$ is well-defined:

$$
\left\langle x^{\prime}, x\right\rangle=\sum_{a \in|X|} x_{a}^{\prime} x_{a}=\sum_{a \in|x| \cap|x|^{\prime}} x_{a}^{\prime} x_{a} \text { is a finite sum. }
$$

The notion of totality, introduced by Girard [8] in denotational semantics, is used for interpreting proofs more closely. It often gives the means to prove completeness results as in Loader [15]. Girard-Loader's totality is described by an orthogonality category up to a slight modification of the partial orthogonality:

$$
u \perp_{\text {tot }} u^{\prime} \Longleftrightarrow \sharp\left(u \cap u^{\prime}\right)=1 .
$$

A totality candidate is then a subset $\Theta(X)$ of $\mathcal{P}(X)$ such that $\Theta(X)$ is $\perp_{\text {tot-closed. A }}$ totality space ${ }^{1}$ is a pair $(|X|, \Theta(X))$ where $\theta(X)$ is a totality candidate.

\footnotetext{
${ }^{1}$ The additional conditions that are actually required in [15] are not essential for our purpose.
} 
The notion of totality can be adapted to linear spaces. So, we introduce the polar orthogonality:

$$
x \perp^{\bullet} x^{\prime} \Longleftrightarrow\left\langle x^{\prime}, x\right\rangle=1 .
$$

Because we are working in a linear algebra setting, we are able to give a simple characterisation of totality candidates, that is polar-closed subspaces of linear finiteness spaces: a totality candidate of a finiteness space is either the space itself, or the empty set, or any topologically closed affine subspace that does not contain 0 and which is topologically closed. We get an orthogonality category whose objects are pairs of a finiteness space and a totality candidate; and whose maps are continuous linear functions that preserve the totality candidates. This is a model of LL.

Since totality candidate are affine spaces, it is natural to add an affine construction to LL: we thus introduce barycentric $L L$. We address then the question of completeness: is it the case that any vector in the totality candidate of a formula is the interpretation of a proof of this formula? Restricting our attention to a barycentric version of the simply typed lambda-calculus (extended with booleans $\mathcal{B}$ and a conditional operator), we prove completeness at type $\mathcal{B}^{n} \Rightarrow \mathcal{B}$ for all $n$, by an algebraic method.

Outline. We start Section 1 with generalities on finiteness spaces at both levels. Then, we give several properties inherited from linearly topologised spaces, in particular, we introduce separation results that are fundamental in the sequel. We describe the interpretation of linear logic proofs into finiteness spaces relying on Ehrhard's results $[6,5]$. In Section 2, after having defined totality candidates and the associated total orthogonality category, we study barycentric $\lambda$-calculus. Finally, in Section 3 , we tackle the completeness problem and give a positive answer for first order boolean types.

\section{Finiteness spaces}

\subsection{Relational finiteness spaces}

Let $\mathbb{A}$ be a countable set. The finite orthogonality is defined by:

$$
\forall u, u^{\prime} \in \mathbb{A}, u \perp u^{\prime} \Longleftrightarrow u \cap u^{\prime} \text { finite. }
$$

As usual, the orthogonal of any $\mathcal{F} \subseteq \mathcal{P}(\mathbb{A})$ is $\mathcal{F}^{\perp}=\left\{u^{\prime} \subseteq \mathbb{A} \mid \forall u \in \mathcal{F}, u \perp u^{\prime}\right\}$ and $\mathcal{F}$ is orthogonally closed whenever $\mathcal{F}^{\perp \perp}=\mathcal{F}$.

Definition 1. A relational finiteness space is a pair $A=(|A|, \mathcal{F}(A))$ where the web $|A|$ is a countable set and the finitary subsets $\mathcal{F}(A) \subseteq \mathcal{P}(|A|)$ are orthogonally closed. We call $u \in \mathcal{F}\left(A^{\perp}\right)$ antifinitary. Let $A$ and $B$ be relational finiteness spaces. A finitary relation $R$ between $A$ and $B$ is a subset of $A \times B$ such that

$$
\begin{aligned}
\forall u \in \mathcal{F}(A), R \cdot u & =\{b \in|B| \mid \exists a \in u,(a, b) \in R\} \in \mathcal{F}(B), \\
\forall v^{\prime} \in \mathcal{F}(B)^{\perp},{ }^{t} R \cdot v^{\prime} & =\left\{a \in|A| \mid \exists b \in v^{\prime},(a, b) \in R\right\} \in \mathcal{F}(A)^{\perp} .
\end{aligned}
$$

Let us call RelFin the category whose objects are the relational finiteness spaces and whose maps are the finitary relations. 
Every finite subset of a countable set $\mathbb{A}$ is finitary. Therefore, there is only one relational finiteness space associated with a finite web (any subset is finitary).

Let $\mathcal{F}, \mathcal{G} \subseteq \mathcal{P}(\mathbb{A})$. If $\mathcal{F} \subseteq \mathcal{G}$ then $\mathcal{G}^{\perp} \subseteq \mathcal{F}^{\perp}$. Besides, $\mathcal{F} \subseteq \mathcal{F}^{\perp \perp}$, hence $\mathcal{F}^{\perp \perp \perp}=$ $\mathcal{F}^{\perp}$. Therefore, $\left(\mathbb{A}, \mathcal{F}^{\perp \perp}\right)$ is always a finiteness space.

Let $A$ be a relational finiteness space, then $\left(\mathcal{F}(A)^{\perp}\right)^{\perp}=\mathcal{F}(A)$. Thus, the orthogonal $A^{\perp}$ of $A$ defined to be $\left(|A|, \mathcal{F}(A)^{\perp}\right)$ is a relational finiteness space whose orthogonal $A^{\perp \perp}=\left(|A|, \mathcal{F}(A)^{\perp \perp}\right)$ is equal to $A$.

Example 2. Booleans. The relational finiteness space $\mathcal{B}$ is associated with the web with two elements $\mathbb{B}=\{\mathrm{T}, \mathrm{F}\}$. Every subset is finitary: $\mathcal{F}(\mathcal{B})=\mathcal{P}(\mathbb{B})$.

Integers. The web $\mathbb{N}$ of integers, associated with the finite subsets $\mathcal{P}_{\text {fin }}(\mathbb{N})$ constitutes a relational finiteness space denoted $\mathcal{N}$. Its orthogonal $\mathcal{N}^{\perp}$ is $(\mathbb{N}, \mathcal{P}(\mathbb{N}))$.

\subsection{Linear finiteness spaces}

Notations: In the sequel $A,\left(A_{i}\right)_{i<n}$ and $B$ range over relational finiteness spaces. The field $\mathbb{k}$ is discrete and infinite (i.e. every subset of $\mathbb{k}$ is open). We handle standard notions of linear algebra using the notations: $\quad E^{*}$ is the space of linear forms over $E, \quad E^{\prime}$ is the topological dual of $E,\left\langle x^{*}, x\right\rangle$ is $x^{*}(x)$ if $x \in E$ and $x^{*} \in E^{*}, \operatorname{ker}_{E}\left(x^{*}\right)$ is the kernel of $x^{*} \in E^{*}, \quad \operatorname{ann}_{E^{*}}(x)$ (resp. ann $E^{\prime}(x)$ ) is the subspace of $E^{*}$ (resp. $\left.E^{\prime}\right)$ of linear forms (resp. continuous linear forms) which annihilate $x$, aff $(D)$ (resp. $\overline{\text { aff }}(D)$ ) is the affine hull (resp. affine closed) of a subset $D$.

Any relational finiteness space $A$ gives rise to a linear finiteness spaces $\mathbb{k}_{k}\langle A\rangle$ which is a subspace of the linear space $\mathbb{k}^{|A|}$ :

Definition 3. For every $x \in \mathbb{k}^{|A|}$, let $|x|=\left\{a \in|A| \mid x_{a} \neq 0\right\}$ be the support of $x$. The linear finiteness space associated with $A$ is $\mathbb{k}\langle A\rangle=\left\{x \in \mathbb{k}^{|A|}|| x \mid \in \mathcal{F}(A)\right\}$.

With each $a \in|A|$, we associate a basic vector $e_{a} \in \mathbb{k}\langle A\rangle$. Notice that $\mathbb{k}\langle A\rangle$ is generated by the finitary linear combinations of basic vectors (and not by finite ones).

Each linear finiteness space can be endowed with a topology which is induced by the antifinitary parts of the underlying relational finiteness space:

Definition 4. For every $J^{\prime} \in \mathcal{F}(A)^{\perp}$, let us call $V_{J^{\prime}}=\left\{x \in \mathbb{k}\langle A\rangle|| x \mid \cap J^{\prime}=\emptyset\right\}$ a fundamental linear neighbourhood of 0 . A subset $U$ of $\mathbb{k}\langle A\rangle$ is open if and only if for each $x \in U$ there is $J_{x}^{\prime} \in \mathcal{F}(A)^{\perp}$ such that $x+V_{J_{x}^{\prime}} \subseteq U$. This topology is named the finiteness topology on $\mathbb{k}\langle A\rangle$.

The collection of $V_{J^{\prime}}$ where $J^{\prime}$ ranges over $\mathcal{F}(A)^{\perp}$ is a filter basis. Indeed, for every $J_{1}^{\prime}, J_{2}^{\prime} \in \mathcal{F}(A)^{\perp}, V_{J_{1}^{\prime}} \cap V_{J_{2}^{\prime}}=V_{J_{1}^{\prime} \cup J_{2}^{\prime}}$ and $J_{1}^{\prime} \cup J_{2}^{\prime} \in(\mathcal{F}(A))^{\perp}$. Besides, $\mathbb{k}\langle A\rangle$ is Hausdorff, since for every $x \neq 0$ and $a \in|x|$ the finite set $\{a\} \in \mathcal{F}(A)^{\perp}$, so $x \notin V_{\{a\}}$.

Endowed with the finiteness topology, $\mathbb{k}\langle A\rangle$ is a linearly topologised space. That is a topological vector space over a discrete field whose topology is generated by a fundamental system (a filter basis of neighbourhoods of 0 , here the $V_{J^{\prime}}$, which are linear subspaces of $\mathbb{k}\langle A\rangle$ ). Introduced by Lefschetz [14, II - §6], linearly topologised spaces have been widely studied in [12, §10-13].

Definition 5. Let us call LinFin the category whose objects are the linear finiteness spaces and whose maps are the linear continuous functions. 
Example 6. Booleans. As every linear finiteness space whose web is finite, the linear finiteness space associated with the boolean relational space has a finite dimension: $\mathbb{k}\langle\mathcal{B}\rangle=\mathbb{k}_{k}\left\langle\mathcal{B}^{\perp}\right\rangle=\mathbb{k}^{\mathbb{B}} \simeq \mathbb{k}^{2}$. The space $\mathbb{k}\langle\mathcal{B}\rangle$ is endowed with the discrete topology since $\mathcal{B}$ is antifinitary and so $V_{\mathcal{B}}=\{0\}$ is a fundamental linear neighbourhood of 0 .

Integers. The linear finiteness space associated with $\mathcal{N}$ is the set of finite sequences over $\mathbb{k}$. The linear finiteness space associated with $\mathcal{N}^{\perp}$ is the set of all sequences over $\mathbb{k}$. Since $\mathbb{N} \in \mathcal{F}\left(\mathcal{N}^{\perp}\right), V_{\mathbb{N}}=\{0\}$ is a neighbourhood of zero, $\mathbb{k}\langle\mathcal{N}\rangle$ is endowed with the discrete topology. On the contrary, the topology on $\mathbb{k}\left\langle\mathcal{N}^{\perp}\right\rangle$ is non-trivial: the fundamental system is the collection of $V_{J^{\prime}}$ where $J^{\prime}$ ranges over finite subsets of $\mathbb{N}$. The space $\mathbb{k}\left\langle\mathcal{N}^{\perp}\right\rangle$ is simply $\mathbb{k}^{\mathbb{N}}$ endowed with the usual product topology.

Linearly topologised spaces are quite different from Banach spaces. Any open subset $V_{J^{\prime}}$ of a finiteness space is closed $\left(\forall x \notin V_{J^{\prime}},\left(x+V_{J^{\prime}}\right) \cap V_{J^{\prime}}=\emptyset\right)$ - linear finiteness spaces are totally disconnected. Intuitively, unit balls are replaced by subspaces, the $V_{J^{\prime}} \mathrm{s}$, which can hardly be considered as bounded in the usual meaning. However, there are linear variants of the classical notions of boundedness and compactness:

Definition 7. A subspace $C$ of $\mathbb{k}\langle A\rangle$ is said linearly bounded iff for every $J^{\prime} \in \mathcal{F}(A)^{\perp}$ the codimension of $V_{J^{\prime}} \cap C$ in $C$ is finite, i.e. there exists a subspace $C_{0}$ of $C$ such that:

$$
C=\left(V_{J^{\prime}} \cap C\right) \oplus C_{0} \text { and } \operatorname{dim} C_{0} \text { is finite. }
$$

A subspace $K$ of $\mathbb{k}\langle A\rangle$ is said linearly compact iff for every filter $\mathcal{F}$ of affine closed subspaces of $\mathbb{k}\langle A\rangle$, which satisfies the intersection property (i.e. $\forall F \in \mathcal{F}, F \cap K \neq \emptyset$ ),

$$
(\cap \mathcal{F}) \cap K \neq \emptyset \text {. }
$$

Theorem 8 (Tychonov). [12, \$10.9(7)] For any set $I, \mathbb{k}^{I}$ endowed with the product topology (generated by $V_{J}=\left\{x \in \mathbb{k}^{I}|| x \mid \cap J=\emptyset\right\}$ with $J \subseteq I$ ) is linearly compact.

In the converse direction, we get a characterisation of linearly compact spaces:

Theorem 9. [14, II - $\$ 6(32.1)]$ For every linearly compact vector space $K$, there is a set I such that $K$ is topologically isomorphic to $\mathbb{k}^{I}$ endowed with the product topology.

Example 10. Booleans. As in every linearly topologised space of finite dimension (see $[12, \S 13.1])$, every subspace of $\mathbb{k}\langle\mathcal{B}\rangle$ is linearly bounded.

Integers. It follows from Th. 9 that a linearly compact space is discrete iff its dimension is finite. Hence, the linearly compact subspaces of $\mathbb{k}\langle\mathcal{N}\rangle$ are the finite dimensional ones. Thanks to Tychonov Th. $8, \mathbb{k}\left\langle\mathcal{N}^{\perp}\right\rangle$ is linearly compact.

In the finiteness setting, finitary support characterise linearly bounded spaces. Although it is not true in the general setting of linearly topologised spaces [12, §13.1(5)], linearly compact spaces are exactly the closed linearly bounded spaces.

Proposition 11. Let $K$ be a subspace of $\mathbb{k}\langle A\rangle$. There is an equivalence between
1. $K$ is linearly bounded,
2. $|K|=\cup\{|x| \mid x \in K\}$ is finitary,

3. the closure of $K$ is linearly compact. 
Proof. First, let $C$ be a linearly bounded space and $J^{\prime} \in \mathcal{F}(A)^{\perp}$. There is a finite dimensional subspace $C_{0}$ of $C$ such that $C=\left(C \cap V_{J^{\prime}}\right) \oplus C_{0}$. Since the dimension of $C_{0}$ is finite, $\left|C_{0}\right|$ is finitary. Besides, $|C| \cap J^{\prime}=\left|C_{0}\right| \cap J^{\prime}$ which is finite: $|C| \in \mathcal{F}(A)$. Conversely, if $|C| \in \mathcal{F}(A)$, then $|C| \cap J^{\prime}$ is finite and $C \subseteq\left(\mathbb{k}^{|K|} \cap V_{J^{\prime}}\right) \oplus \mathbb{k}^{|K| \cap J^{\prime}}$ is linearly bounded. The equivalence between (2) and (3) has already been proved in [5]. (Complete proof in Annex A, Prop. 11)

We focus attention on the topological dual - a linearly topologised space endowed with the compact open topology, that is the topology of uniform convergence on either linearly bounded spaces or linearly compact spaces (equivalent thanks to Prop. 11).

Definition 12. The topological dual $\mathbb{k}\langle A\rangle^{\prime}$ is the linear space made of continuous linear forms over $\mathbb{k}\langle A\rangle$ and endowed with the linearly compact open topology. This topology is generated by the $\operatorname{ann}_{\mathbb{k}\langle A\rangle^{\prime}}(K)=\left\{x^{\prime} \in \mathbb{k}\langle A\rangle^{\prime} \mid \forall x \in K,\left\langle x^{\prime}, x\right\rangle=0\right\}$ s where $K$ ranges over linearly compact subspaces of $\mathbb{k}\langle A\rangle$.

The two following propositions are central in the totality introduced in Section 2.

Proposition 13 (Separation). [12, $\left.\$ 10.4\left(1^{\prime}\right)\right]$. For every closed subspace D of $\mathbb{k}_{k}\langle A\rangle$ and $x \notin D$, there is a continuous linear form $x^{\prime} \in \mathbb{k}\langle A\rangle^{\prime}$ such that $\left\langle x, x^{\prime}\right\rangle=1$ and $\forall d \in D,\left\langle d, x^{\prime}\right\rangle=0$.

(Proof in Annex A, Prop. 13)

Proposition 14 (Separation in the dual). Let $T^{\prime}$ be a closed affine subspace of $\mathbb{k}\langle A\rangle^{\prime}$ such that $0 \notin T^{\prime}$. There exists $x \in \mathbb{k}\langle A\rangle$ such that $\forall x^{\prime} \in T^{\prime},\left\langle x^{\prime}, x\right\rangle=1$.

Proof. First, we linear algebra ensures the result when the dimension of $T^{\prime}$ is finite (c.f. Annex A, Lem.14). Second, the closed subspace $T^{\prime}$ does not contain 0, so there is $K$ linearly compact of $\mathbb{k}\langle A\rangle$ such that ann $(K) \cap T^{\prime}=\emptyset$. We use the closed affine filter made of $T_{F^{\prime}}=\left\{x \in E \mid \forall x^{\prime} \in F^{\prime},\left\langle x^{\prime}, x\right\rangle=1\right\}$ with $F^{\prime}$ ranging over finite collections of $\mathbb{k}\langle A\rangle^{\prime}$ and the compactness of $K$ to build the wanted $x$. (Details in Annex A, Prop. 14)

Both separations theorem, ensure the algebraic isomorphism between a linear finiteness space and its second dual. This isomorphism is both continuous and open, as linearly bounded subspaces of the dual coincide with equicontinuous subspaces (Prop. 15). To sum up, the reflexivity of finiteness spaces relies on the links between linearly compactness, closed linearly bounded and equicontinuity.

(Annex A, Prop 16-17)

Proposition 15. (Equicontinuous spaces) Let $A$ be a relational finiteness space. A subspace $B^{\prime}$ of $\mathbb{k}\langle A\rangle^{\prime}$ is linearly bounded if and only if there is $J^{\prime} \in \mathcal{F}(A)^{\perp}$ such that $B^{\prime} \subseteq \operatorname{ann}_{\mathbb{k}\langle A\rangle^{\prime}}\left(V_{J^{\prime}}\right)$.

(proof in Annex A, Prop. 15)

Linear finiteness spaces satisfy other good properties (they admit Schauder bases $\left(e_{a}\right)_{a \in|A|}$ and are complete [6]). Although we do not know by now if the category of linearly topologised spaces satisfying all these properties is stable under LL constructions, we already know that the full subcategory of finiteness spaces is a model of LL. 


\subsection{A model of MELL with MIX}

Both categories RelFin and LinFin constitute a model of classical linear logic as it has been proved by Ehrhard [6]. Although linear finiteness spaces are entirely determined by their underlying relational finiteness space (Fig.1), we give the algebraic description of the constructions of LL in LinFin (Fig.2) as in [6,5].

$$
\begin{aligned}
& \text { Multiplicatives: } \\
& |A \Gamma B|=|A \otimes B|=|A| \times|B| \\
& \mathcal{F}(A \Gamma B)=\left\{\begin{array}{l}
R \subseteq|A| \times|B| \text { s.t. } \\
\forall u \in \mathcal{F}(A)^{\perp}, R \cdot u \in \mathcal{F}(B) \\
\forall v \in \mathcal{F}(B)^{\perp},{ }^{t} R \cdot v \in \mathcal{F}(A)
\end{array}\right\} \\
& \mathcal{F}(A \otimes B)=\left\{\begin{aligned}
R \subseteq|A| \times|B| \text { s.t. } \\
R \cdot|B| \in \mathcal{F}(A) \\
{ }^{t} R \cdot|A| \in \mathcal{F}(B)
\end{aligned}\right\} \\
& \mathcal{F}\left(\&_{i} A_{i}\right)=\left\{\begin{array}{l}
\sqcup_{i} u_{i} \text { s.t. } \\
\forall i \in I, u_{i} \in \mathcal{F}\left(A_{i}\right)
\end{array}\right\} \\
& \mathcal{F}\left(\oplus_{i} A_{i}\right)=\left\{\begin{array}{c}
\sqcup_{j \in J} u_{j} \text { s.t. } \\
J \subseteq I \text { finite } \\
\forall j \in J, u_{j} \in \mathcal{F}\left(A_{j}\right)
\end{array}\right\} \\
& |! A|=|? A|=\mathcal{M}_{\text {fin }}(|A|)=\{\mu: \mathbb{A} \rightarrow \mathbb{N} \mid \mu(a)>0 \text { for finitely many } a \in A\} \\
& \mathcal{F}(! A)=\left\{M \subseteq \mathcal{M}_{\text {fin }}(|A|) \mid \cup\{|\mu|, \mu \in M\} \in \mathcal{F}(A)\right\} \\
& \mathcal{F}(? A)=\left\{M \subseteq \mathcal{M}_{\text {fin }}(|A|) \mid \forall u \in \mathcal{F}(A)^{\perp}, \mathcal{M}_{\text {fin }}(u) \cap M \text { finite }\right\}
\end{aligned}
$$

\section{Exponentials:}

Fig. 1: LL interpreted in RelFin; proofs are interpreted as in the relational model.

Example 16. If $\mathcal{B}=1 \oplus 1$, then we get back to Ex. $2: \llbracket \mathcal{B} \rrbracket=\mathbb{k} \oplus \mathbb{k} \simeq \mathbb{k}^{2}$.

$$
\begin{gathered}
\left|? \mathcal{B}^{\perp}\right|=|! \mathcal{B}|=\mathcal{M}_{\text {fin }}(\mathrm{T}, \mathrm{F}) \simeq \mathbb{N}^{2}, \\
\mathcal{F}(! \mathcal{B})=\left\{M \subseteq \mathcal{M}_{\text {fin }}(\mathrm{T}, \mathrm{F})\left|\cup_{\mu \in M}\right| \mu \mid \in \mathcal{F}(\mathcal{B})\right\}=\mathcal{P}\left(\mathbb{N}^{2}\right), \\
\mathcal{F}\left(? \mathcal{B}^{\perp}\right)=\left\{M \subseteq \mathbb{N}^{2} \mid \forall M^{\prime} \subseteq \mathbb{N}^{2}, M \cap M^{\prime} \text { fin. }\right\}=\mathcal{P}_{\text {fin }}\left(\mathbb{N}^{2}\right) .
\end{gathered}
$$

\begin{tabular}{|l|l|}
\hline Multiplicatives: & Additives: \\
$\mathbb{k}\langle\perp\rangle=\mathbb{k}\langle 1\rangle=\mathbb{k}$ & $\mathbb{k}\langle T\rangle=\mathbb{k}\langle 0\rangle=\{0\}$ \\
$\mathbb{k}\langle A \Gamma B\rangle=\mathbb{k}\langle A\rangle \otimes_{\varepsilon} \mathbb{k}\langle B\rangle$ & $\mathbb{k}\left\langle \&_{i \in I} A_{i}\right\rangle=\times_{i \in I} \mathbb{k}\langle A\rangle_{i}$ \\
$\mathbb{k}\langle A \otimes B\rangle=\mathbb{k}\langle A\rangle \widetilde{\otimes} \mathbb{k}\langle B\rangle$ & $\mathbb{k}\left\langle\oplus_{i \in I} A_{i}\right\rangle=\oplus_{i \in I} \mathbb{k}\langle A\rangle_{i}$ \\
$\mathbb{k}\langle A \multimap B\rangle=\mathcal{L}_{\mathrm{c}}(\mathbb{k}\langle A\rangle, \mathbb{k}\langle B\rangle)$ & \\
\hline \multicolumn{1}{|c|}{ Exponentials: } \\
$\mathbb{k}\left\langle ? A^{\perp}\right\rangle=\widetilde{\operatorname{Pol}}(\mathbb{k}\langle A\rangle)$ & $\mathbb{k}\langle! A\rangle=[\widetilde{\operatorname{Pol}}(\mathbb{k}\langle A\rangle)]^{\prime}$
\end{tabular}

Fig. 2: Interpretation of formulæ in LinFin, for proofs, see Annex B. 
Let us give some explanations on Fig.2:

$(\rightarrow)$ continuous linear functions. We can generalise the topological dual framework and endow the space of continuous linear functions $\mathcal{L}_{\mathrm{c}}\left(\mathbb{k}_{\mathrm{k}}\langle A\rangle, \mathbb{k}_{\mathrm{k}}\langle B\rangle\right)$ with the linearly compact open topology. It is generated by $\mathcal{W}(K, V)=\{f \mid f(K) \subseteq V\}$ where $K$ ranges over linearly compact subspaces of $\mathbb{k}\langle A\rangle$ and $V$ over fundamental neighbourhoods of 0 of $\mathbb{k}\langle B\rangle$.

The linearly topologised space $\mathcal{L}_{\mathrm{c}}(\mathbb{k}\langle A\rangle, \mathbb{k}\langle B\rangle)$ coincides with the linear finiteness space $\mathbb{k}\langle A \multimap B\rangle=\mathbb{k}\left\langle A^{\perp} \Gamma B\right\rangle$. Indeed, the canonical map which maps each linear function to its matrix in the base induced by the web is a linear homeomorphism.

$(\Gamma)$ hypocontinuous bilinear forms. As noticed by Ehrhard, the evaluation map: ev $: \mathbb{k}\langle A \multimap B\rangle \times \mathbb{k}\langle A\rangle \rightarrow \mathbb{k}\langle B\rangle$ is separately continuous but not continuous. That is why we need another the notion of hypocontinuity:

A bilinear form $\phi: \mathbb{k}\langle A\rangle \times \mathbb{k}\langle B\rangle \rightarrow \mathbb{k}$ is said hypocontinuous iff for every linearly compacts $K_{A}$ of $\mathbb{k}\langle A\rangle$ and $K_{B}$ of $\mathbb{k}\langle B\rangle$, there are two neighbourhoods $V_{B}$ of $\mathbb{k}\langle B\rangle$ and $V_{A}$ of $\mathbb{k}\langle A\rangle$ such that $\phi\left(K_{A}, V_{B}\right)=0$ and $\phi\left(V_{A}, K_{B}\right)=0$. We denote $\mathbb{k}\langle A\rangle \otimes_{\varepsilon} \mathbb{k}\langle B\rangle$, the space of hypocontinuous bilinear forms over $\mathbb{k}\langle A\rangle^{\prime} \times \mathbb{k}\langle B\rangle^{\prime}$. It is a linearly topologised space when it is endowed with the linearly compact open topology generated by $\mathcal{W}\left(K_{A}^{\prime}, K_{B}^{\prime}\right)=\left\{\phi \mid \phi\left(K_{A}^{\prime}, K_{B}^{\prime}\right)=0\right\}$ where $K_{A}^{\prime}$ and $K_{B}^{\prime}$ range over linearly compact subspaces of $\mathbb{k}\langle A\rangle^{\prime}$ and $\mathbb{k}\langle B\rangle^{\prime}$ respectively.

The space $\mathbb{k}\langle A\rangle \otimes_{\varepsilon} \mathbb{k}\langle B\rangle$ is related to the inductive tensor product [10] which was generalised to linearly topologised space in [7].

$(\otimes)$ complete tensor product. The dual $\mathbb{k}\langle A\rangle \widetilde{\otimes} \mathbb{k}\langle B\rangle$ of $\mathbb{k}\langle A\rangle^{\prime} \otimes_{\varepsilon} \mathbb{k}\langle B\rangle^{\prime}$ is the completion of the algebraic tensor product $\mathbb{k}\langle A\rangle \otimes \mathbb{k}\langle B\rangle$. Indeed, $\alpha(\mathbb{k}\langle A\rangle \otimes \mathbb{k}\langle B\rangle)$ is dense in $\mathbb{k}\langle A\rangle \widetilde{\otimes} \mathbb{k}\langle B\rangle[7$, Th 2.12] where

$$
\begin{aligned}
\alpha: \mathbb{k}\langle A\rangle \otimes \mathbb{k}\langle B\rangle & \hookrightarrow\left(\mathbb{k}\langle A\rangle^{\prime} \otimes_{\varepsilon} \mathbb{k}\langle B\rangle^{\prime}\right)^{\prime} \\
x \otimes y & \mapsto[x \widetilde{\otimes} y: \phi \mapsto \phi(x, y)] .
\end{aligned}
$$

(\&) product. Let $I$ be a set. The linear finiteness space $\mathbb{k}\left\langle \&_{i \in I} A_{i}\right\rangle$ is the product of the $\mathbb{k}\langle A\rangle_{i} \mathrm{~s}$, endowed with the product topology. $(\oplus)$ direct sum. The linear finiteness space $\mathbb{k}\left\langle\oplus_{i \in I} A_{i}\right\rangle$ is the coproduct $\oplus_{i \in I} \mathbb{k}\langle A\rangle_{i}$ (made of finite linear combinations of elements of the $\mathbb{k}\langle A\rangle_{i} \mathrm{~s}$ ), endowed with the topology induced by the product topology.

(!) through webs. The comonadic structure $(\mathbb{k}\langle! A\rangle, \epsilon, \delta)$ and its linear distribution $\kappa$, can be described with respect to the web base: for $x=\sum_{a \in|A|} x_{a} e_{A}$ given in $\mathbb{k}\langle A\rangle$, we set $x^{\mu}=\prod_{a \in|a|} x_{a}^{\mu(a)}$ and we take $X=\sum_{\mu \in \mathcal{M}_{\mathrm{fin}}(|A|)} x_{\mu} e_{\mu} \in \mathbb{k}\langle! A\rangle$ in

$$
\begin{gathered}
\kappa: x \mapsto \sum_{\mu \in \mathcal{M}_{\mathrm{fin}}(|A|)} x^{\mu} e_{\mu} \quad \epsilon: X \mapsto \sum_{a \in|A|} x_{[a]} e_{a}, \\
\delta: X \mapsto \sum_{M \in \mathcal{M}_{\mathrm{fin}}\left(\mathcal{M}_{\mathrm{fin}}(|A|)\right)}\left(\sum_{\mu=\Sigma(M)} x_{\mu}\right) e_{M} .
\end{gathered}
$$

The exponentiation $x^{!}=\kappa(x)$ of $x$ satisfies $\epsilon\left(x^{!}\right)=x$ and $\delta\left(x^{!}\right)=\left(x^{!}\right)^{!}$.

(?) analytic functions. The linear finiteness space $\mathbb{k}\left\langle ? A^{\perp}\right\rangle$ is the dual of $\mathbb{k}\langle! A\rangle$. However, there is a more algebraic approach [5] of the monoid $\mathbb{k}\left\langle ? A^{\perp}\right\rangle$. A function $P$ is polynomial whenever there are symmetric hypocontinous $i$-linear forms ${ }^{2} \phi_{i}$ :

\footnotetext{
${ }^{2}$ Hypocontinuity for $i$-linear forms is a generalisation of the bilinear case. (Annex B, Def. 18)
} 
$\times^{i} \mathbb{k}\langle A\rangle^{\prime} \rightarrow \mathbb{k}$ such that $P(x)=\sum_{i=0}^{n} \phi_{i}(x, \ldots, x)$. The space $\mathbb{k}\left\langle ? A^{\perp}\right\rangle$ coincides with the completion of polynomial functions over $\mathbb{k}\langle A\rangle$ endowed with the linearly compact open topology ${ }^{3}$. We call the elements of $\mathbb{k}\left\langle ? A^{\perp}\right\rangle$ analytic functions.

(!) distributions. Finally, we are concerned with the Taylor expansion formula of Ehrhard [6]. Taking into account that $\mathbb{k}\langle! A\rangle$ is the dual space of $\widetilde{\operatorname{Pol}}(\mathbb{k}\langle A\rangle)$, we can establish a parallel with distributions. For instance, $x^{!}$sends an analytic function $F$ to its image $\left\langle x^{!}, F\right\rangle=F(x)$, hence it corresponds to the dirac mass at $x$. Besides, in LinFin, there exists a sequence of projections:

$$
\pi_{n}: \sum_{\mu \in \mathcal{M}_{\mathrm{fin}}(|A|)} x_{\mu} e_{\mu} \in \mathbb{k}\langle! A\rangle \mapsto \sum_{\sharp \mu=n} x_{\mu} e_{\mu} \in \mathbb{k}\langle! A\rangle,
$$

which are linear and continuous since their support $|\pi|_{n}=\{(\mu, \mu) \mid \sharp \mu=n\}$ are finitary. The vector $x^{n}=\pi_{n}\left(x^{!}\right)=\sum_{\sharp \mu=n} x^{\mu} e_{\mu}$ of $\mathbb{k}\langle! A\rangle$ is the convolution of $x$ iterated $n$ times. This distribution sends an analytic function to a homogeneous polynomial of degree $n$, that is its derivative at zero. From $x^{!}=\sum_{n=0}^{\infty} \frac{1}{n !} x^{n}$, Ehrhard deduces the Taylor expansion formula:

$$
F(x)=\sum_{n=0}^{\infty} \frac{1}{n !}\left\langle x^{n}, F\right\rangle .
$$

Example 17. $\mathbb{k}\langle! \mathcal{B}\rangle=\left\{z \in \mathbb{k}^{\mathbb{N}^{2}}|| z \mid \in \mathcal{P}\left(\mathbb{N}^{2}\right)\right\}=\mathbb{k}\left(X_{t}, X_{f}\right)$

$\mathbb{k}\left\langle ? \mathcal{B}^{\perp}\right\rangle=\left\{z \in \mathbb{k}^{\mathbb{N}^{2}}|| z \mid \in \mathcal{P}_{\text {fin }}\left(\mathbb{N}^{2}\right)\right\}=\mathbb{k}\left[X_{t}, X_{f}\right]$

$\mathbb{k}\langle! \mathcal{B} \multimap \mathcal{B}\rangle=\mathbb{k}\langle! \mathcal{B} \multimap 1 \oplus 1\rangle=\mathbb{k}\left\langle ? \mathcal{B}^{\perp}\right\rangle^{2}=\mathbb{k}\left[X_{t}, X_{f}\right] \times \mathbb{k}\left[X_{t}, X_{f}\right]$,

$\mathbb{k}\left\langle\Gamma^{n} ? \mathcal{B}^{\perp}\right\rangle=\mathbb{k}\left[X_{1}, X_{2}, \ldots, X_{2 n-1}, X_{2 n}\right]$

$\mathbb{k}\left\langle\otimes^{n} ! \mathcal{B} \multimap \mathcal{B}\right\rangle=\mathbb{k}\left[X_{1}, X_{2}, \ldots, X_{2 n-1}, X_{2 n}\right]^{2}$

\section{Totality and Barycentric lambda-calculus}

In the present section, we explore an algebraic version of totality spaces, where formulæ are interpreted as finiteness spaces with an additional totality structure. Adapting Loader's definition to this algebraic setting, we define a general concept of totality finiteness space: it is a pair $[\mathbb{k}\langle A\rangle, \mathcal{T}]$ where $\mathbb{k}\langle A\rangle$ is a linear finiteness space and $\mathcal{T}$ is a subset of $\mathbb{k}\langle A\rangle$ which is equal to its second dual for a duality associated with the polar as defined below. Actually, the finiteness space interpreting any formula coincides with the first component of the totality finiteness space interpreting this formula.

Notations: $\operatorname{dir}(T)$ is the direction of the affine space $T$ and if $x \in T, T=x+\operatorname{dir}(T)$, $f^{*}: y^{*} \in F^{*} \mapsto\left[x^{*} \in E^{*}: x \mapsto\left\langle y^{*}, f(x)\right\rangle\right]$ is the linear adjoint of $f: E \rightarrow F$. $A$ and $B$ are relational finiteness spaces.

\subsection{Totality finiteness spaces.}

The polar orthogonality is defined as follows:

$$
\forall x \in \mathbb{k}\langle A\rangle, x^{\prime} \in \mathbb{k}\langle A\rangle^{\prime}, x \perp \cdot x^{\prime} \Longleftrightarrow\left\langle x^{\prime}, x\right\rangle=1
$$

\footnotetext{
${ }^{3}$ generated by $\mathcal{W}(K)=\{P$ polynomial function $\mid P(K)=0\}$ with $K$ linearly compact.
} 
The polar of a subset $\mathcal{T}$ of $\mathbb{k}\langle A\rangle$ is the following closed affine subspace of $\mathbb{k}\langle A\rangle^{\prime}$ :

$$
\mathcal{T}^{\bullet}=\left\{x^{\prime} \in \mathbb{k}\langle A\rangle^{\prime} \mid \forall x \in \mathcal{T},\left\langle x^{\prime}, x\right\rangle=1\right\}
$$

This set is closed since $\left(x, x^{\prime}\right) \mapsto\left\langle x^{\prime}, x\right\rangle$ is linear and separately continuous on $\mathbb{k}\langle A\rangle \times$ $\mathbb{k}\langle A\rangle^{\prime}$ (let $\left(x, x^{\prime}\right) \in \mathbb{k}\langle A\rangle \times \mathbb{k}\langle A\rangle^{\prime},\{x\}$ is linearly compact, so ann $(x)$ is open in $\mathbb{k}\langle A\rangle^{\prime} ; x^{\prime}$ is a linear continuous form, so $\operatorname{ker}\left(x^{\prime}\right)$ is open in $\left.\mathbb{k}\langle A\rangle\right)$. Notice that, up to the homeomorphism between $\mathbb{k}\langle A\rangle$ and $\mathbb{k}\langle A\rangle^{\prime \prime}$, if $\mathcal{T}^{\prime}$ is an affine subspace of $\mathbb{k}\langle A\rangle^{\prime}$, $\mathcal{T}^{\prime \bullet}=\left\{x \in \mathbb{k}\langle A\rangle \mid \forall x^{\prime} \in \mathcal{T}^{\prime},\left\langle x^{\prime}, x\right\rangle=1\right\}$.

There is a simple characterisation of polar-closed affine subspaces:

Proposition 18 (Characterisation). A subspace $\mathcal{T}$ of $\mathbb{k}\langle A\rangle$ is polar-closed iff it is the empty set, the space $\mathbb{k}\langle A\rangle$, or a closed affine subspace that does not contain 0 .

Proof. If $\mathcal{T}=\mathbb{k}\langle A\rangle$, then $\mathcal{T}^{\bullet}=\emptyset$ and $\emptyset \bullet=\mathbb{k}\langle A\rangle=\mathcal{T}$. If $\mathcal{T}=\emptyset$, then $\mathcal{T} \bullet=\emptyset$. It remains the case where $\mathcal{T}$ is affine, closed and $0 \notin \mathcal{T}$. The inclusion $\mathcal{T} \subseteq \mathcal{T}^{\bullet \bullet}$ is straightforward. Let us prove the contrapositive. Let $x_{0} \notin \mathcal{T}$. Let $z_{0} \in \mathcal{T}$ and $D=$ $\operatorname{dir}(\mathcal{T})$ then $\mathcal{T}=z_{0}+D, x_{0} \neq z_{0}$ and $x_{0}-z_{0} \notin D$. By separation Prop. 13, there is $x_{0}^{\prime} \in \mathbb{k}\langle A\rangle^{\prime}$ such that $\left\langle x_{0}^{\prime}, x_{0}-z_{0}\right\rangle=1$ and $\forall d \in D,\left\langle x_{0}^{\prime}, d\right\rangle=0$. On the one side, if $\lambda=\left\langle x_{0}^{\prime}, z_{0}\right\rangle \neq 0$, we set $y_{0}^{\prime}=\frac{1}{\lambda} x_{0}^{\prime}$, then $\left\langle y_{0}^{\prime}, z_{0}\right\rangle=1$ and $\forall d \in D,\left\langle y_{0}^{\prime}, d\right\rangle=$ $\frac{1}{\lambda}\left\langle x_{0}^{\prime}, d\right\rangle=0$, so $y_{0}^{\prime} \in \mathcal{T}^{\bullet}$. However $\left\langle y_{0}^{\prime}, x_{0}\right\rangle=\frac{1}{\lambda}\left\langle x_{0}, x_{0}^{\prime}\right\rangle=\frac{1+\lambda}{\lambda} \neq 1$, hence $x_{0} \notin$ $\mathcal{T}^{\bullet} \bullet$. On the other side, $\left\langle x_{0}^{\prime}, z_{0}\right\rangle=0$, then $\left\langle x_{0}^{\prime}, x_{0}\right\rangle=1$. Since $0 \notin \mathcal{T}$ and by separation Prop. 13, there exists $x_{1}^{\prime} \in \mathbb{k}\langle A\rangle^{\prime}$ such that $\left\langle x_{1}^{\prime}, z_{0}\right\rangle=1$ and $\forall d \in D,\left\langle x_{1}^{\prime}, d\right\rangle=0$, hence $x_{1}^{\prime} \in \mathcal{T}^{\bullet}$. Moreover, $\left\langle x_{1}^{\prime}+x_{0}^{\prime}, z_{0}\right\rangle=1$ and $\forall d \in D,\left\langle x_{1}^{\prime}+x_{0}^{\prime}, d\right\rangle=0$ hence $x_{1}^{\prime}+x_{0}^{\prime} \in \mathcal{T}^{\bullet}$. To conclude, either $\left\langle x_{1}^{\prime}, x_{0}\right\rangle=0$ and $x_{1}^{\prime} \in \mathcal{T}^{\bullet}$ or $\left\langle x_{0}^{\prime}+x_{1}^{\prime}, x_{0}\right\rangle=$ $1+\left\langle x_{0}, x_{1}^{\prime}\right\rangle \neq 1$ and $x_{0}^{\prime}+x_{1}^{\prime} \in \mathcal{T}^{\bullet}$, so in both cases, $x_{0} \notin \mathcal{T} \bullet \bullet$.

From this characterisation, we deduce another one which will be useful to compute the constructions of the model.

Corollary 19. Let $\mathcal{T}$ be a subset of $\mathbb{k}\langle A\rangle$. If $\mathcal{T} \bullet \neq \emptyset$, then $\mathcal{T} \bullet=\overline{\text { aff }}(\mathcal{T})$.

Proof. The proof is based on: $\mathcal{T} \subseteq \overline{\text { aff }}(\mathcal{T}) \subseteq \mathcal{T} \bullet$.

(Proof in Annex C)

Definition 20. A totality finiteness space is a pair $[\mathbb{k}\langle A\rangle, \mathcal{T}]$ made of a linear finiteness space $\mathbb{k}\langle A\rangle$ and a totality candidate $\mathcal{T}$, that is is a polar closed subspace of $\mathbb{k}\langle A\rangle$.

Let TotFin be the category whose objects are totality finiteness spaces and whose morphisms are continuous linear functions that preserve the totality candidates.

\subsection{A model of classical linear logic}

To prove that TotFin is a model of classical linear logic, we use the definitions and results of [11, §4-5]. Let $\mathcal{G}(\mathbf{L F})$ be the double glueing of the category LinFin along the HOM functor. The objects of $\mathcal{G}(\mathbf{L F})$ are triples $\left[\mathbb{k}\langle A\rangle, U, U^{\prime}\right]$ where $U$ and $U^{\prime}$ are subspaces of respectively $\mathbb{k}\langle A\rangle$ and $\mathbb{k}\langle A\rangle^{\prime}$. A morphism between $\left[\mathbb{k}\langle A\rangle, U, U^{\prime}\right]$ and $\left[\mathbb{k}\langle B\rangle, V, V^{\prime}\right]$ is a continuous linear function $f: \mathbb{k}\langle A\rangle \rightarrow \mathbb{k}\langle B\rangle$ such that $f(U) \subseteq V$ and $f^{*}\left(V^{\prime}\right) \subseteq U^{\prime}$, where $f^{*}$ is the adjoint of $f$. The linear exponential comonad of 
LinFin is equipped with a well-behaved linear distribution $\kappa: x \in \mathbb{k}\langle A\rangle \mapsto x^{!} \in$ $\mathbb{k}\langle! A\rangle$ (it is routine to check the diagrams satisfied by $\kappa$ ).

The category TotFin is a subcategory of $\mathcal{G}(\mathbf{L F})$ (considering triples $[\mathbb{k}\langle A\rangle, \mathcal{T}, \mathcal{T} \bullet]$ ). More precisely, it is a Tight orthogonality with respect to the polar orthogonality. This orthogonality is stable since it is focussed with respect to the focus $\{1\}: x \perp^{\bullet} x^{\prime} \Longleftrightarrow$ $\left\langle x^{\prime}, x\right\rangle=1 \Longleftrightarrow x^{\prime}(x)=1$. Since LinFin is a model of classical linear logic, TotFin is also a model of classical linear logic (c.f. [11, Th. 5.14]).

The constructions inherited from LinFin as described in $[11, \S 5.3]$ are:

$$
\begin{array}{cc}
\mathcal{T}\left(A^{\perp}\right)=\mathcal{T}(A)^{\bullet} \\
\mathcal{T}(1)=\mathcal{T}(\perp)=\{1\}, \quad \mathcal{T}(0)=\mathcal{T}(T)=\{0\}, \\
\mathcal{T}(A \otimes B)=[\mathcal{T}(A) \otimes \mathcal{T}(B)]^{\bullet \bullet}, & \mathcal{T}(A \& B)=\mathcal{T}(A) \times \mathcal{T}(B), \\
\mathcal{T}(A \multimap B)=\left[\mathcal{T}(A) \otimes \mathcal{T}(B)^{\bullet} \bullet \bullet\right. & T(A \oplus B)=[\mathcal{T}(A) \times \mathcal{T}(B)]^{\bullet}, \\
\mathcal{T}(! A)=[\kappa(\mathcal{T}(A))]^{\bullet \bullet}=\left\{x^{!} \mid x \in \mathcal{T}(A)\right\}
\end{array}
$$

Moreover, we describe every totality candidate as a closed affine subspace. This algebraic description is made possible thanks to the characterisation of totality candidate (Prop. 18) and to the algebraic setting.

\section{Proposition 21.}

$$
\begin{aligned}
\mathcal{T}(A \otimes B) & =\overline{\operatorname{aff}}(\mathcal{T}(A) \otimes \mathcal{T}(B)), \\
\mathcal{T}(A \multimap B) & =\{f \in \mathbb{k}\langle A\rangle \mid f(\mathcal{T}(A)) \in \mathcal{T}(B)\} . \\
\mathcal{T}(A \oplus B) & =\overline{\operatorname{aff}}\left(\mathcal{T}(A) \times \operatorname{ker}\left(\mathcal{T}(B)^{\bullet}\right) \cup \operatorname{ker}\left(\mathcal{T}(A)^{\bullet}\right) \times \mathcal{T}(B)\right) \\
\mathcal{T}(! A) & =\overline{\operatorname{aff}}\left(x^{!} \mid x \in \mathcal{T}(A)\right), \\
\mathcal{T}(? A) & =\{F \in \widetilde{\operatorname{Pol}}(\mathbb{k}\langle A\rangle) \mid \forall x \in \mathcal{T}(A), F(x)=1\}, \\
\mathcal{T}(! A \multimap B) & =\{F \in \widetilde{\operatorname{Pol}}(\mathbb{k}\langle A\rangle, B) \mid \forall x \in \mathcal{T}(A), F(x) \in \mathcal{T}(B)\} .
\end{aligned}
$$

Proof. The proof relies on showing that $\mathcal{T}^{\bullet}$ is not empty and to use Cor. 19.

( Details in Annex C, Prop 21-23.)

The formula $A \Rightarrow B$ is interpreted as the totality finiteness space that is made of morphisms of the Kleisli category. The totality candidate associated with $A \Rightarrow B$ satisfies the following fundamental equation:

$$
\mathcal{T}(A \Rightarrow B)=\{F: \mathbb{k}\langle A\rangle \rightarrow \mathbb{k}\langle B\rangle \text { analytic } \mid \forall x \in \mathcal{T}(A), F(x) \in \mathcal{T}(B)\}
$$

In other word, the totality we have defined is a logical relation.

$$
\begin{gathered}
\text { Example 22. } \mathcal{T}\langle\mathcal{B}\rangle=\left\{\left(x_{t}, y_{t}\right) \in \mathbb{k}^{2} \mid x_{t}+y_{t}=1\right\}, \quad \mathcal{T}\left\langle\mathcal{B}^{\perp}\right\rangle=\{(1,1)\}, \\
\mathcal{T}\langle! \mathcal{B}\rangle=\left\{F \in \mathbb{k}\left(X_{t}, X_{f}\right) \mid \forall\left(x_{t}, y_{t}\right), x_{t}+y_{t}=1 \Rightarrow F\left(x_{t}, y_{t}\right)=1\right\}, \\
\mathcal{T}\left\langle\mathcal{B}^{\perp}\right\rangle=\left\{P \in \mathbb{k}\left[X_{t}, X_{f}\right] \mid x_{t}+y_{t}=1 \Rightarrow P\left(x_{t}, y_{t}\right)=1\right\}, \\
\mathcal{T}\langle! \mathcal{B} \multimap \mathcal{B}\rangle=\left\{(P, Q) \in \mathbb{k}\left[X_{t}, X_{f}\right]^{2} \mid x_{t}+y_{t}=1 \Rightarrow P\left(x_{t}, y_{t}\right)+Q\left(x_{t}, y_{t}\right)=1\right\} \\
\mathcal{T}\left\langle\Gamma^{n} ? \mathcal{B}^{\perp}\right\rangle=\left\{P \in \mathbb{k}\left[X_{1}, \ldots, X_{2 n}\right] \mid \forall 1 \leq i \leq n, x_{2 i-1}+x_{2 i}=1\right. \\
\left.\Rightarrow P\left(x_{1}, y_{2}, \ldots, x_{2 n-1}, x_{2 n}\right)=1\right\} \\
\mathcal{T}\left\langle\otimes^{n} ! \mathcal{B} \multimap \mathcal{B}\right\rangle=\left\{(P, Q) \in \mathbb{k}\left[X_{1}, \ldots, X_{2 n}\right]^{2} \mid P+Q-1 \in \mathcal{T}\left\langle\Gamma^{n} ? \mathcal{B}\right\rangle\right\}
\end{gathered}
$$


Because totality candidate are affine spaces, it is natural to add a barycentric construction to our proof system and to interpret it by a barycentric combination. Totality finiteness spaces constitute a model of linear logic with MIX and barycentric sums.

\subsection{Simply typed boolean barycentric lambda-calculus}

We propose a $\lambda$-calculus in the style of Vaux's algebraic $\lambda$-calculus [17]. In the barycentric $\lambda$-calculus, sums of terms are allowed. It is well known that the application in $\lambda$ calculus is linear in the function but not in its argument. That is why we introduce two kinds of terms: atomic terms that do not contain barycentric sums but in the argument of an application and barycentric terms which are barycentric sums of atomic terms. Moreover, we add booleans and a conditional construction.

Syntax. Let $\mathcal{V}$ be a countable set of variables. Atomic terms $\mathbf{s}$ and barycentric terms $\mathbf{T}$ are inductively defined by

$$
\begin{aligned}
& \mathbf{R}, \mathbf{S}::=\sum_{i=1}^{m} a_{i} \mathbf{s}_{i} \quad \text { and }\left\{\begin{array}{l}
\forall i \in\{1, \ldots, m\}, a_{i} \in \mathbb{k}, \\
\sum_{i=1}^{m} a_{i}=1,
\end{array}\right. \\
& \mathbf{s}::=x|\lambda x . \mathbf{s}|(\mathbf{s}) \mathbf{T}|\mathrm{T}| \mathrm{F} \mid \text { if } \mathbf{s} \text { then } \mathbf{S} \text { else } \mathbf{R} \quad \text { where } \quad x \in \mathcal{V}
\end{aligned}
$$

We denote $\boldsymbol{\Lambda}_{\text {at }}$ the collection of atomic terms and $\boldsymbol{\Lambda}_{\text {bar }}$ the collection of barycentric terms. We quotient all these sets of terms by $\alpha$-conversion and associativity and commutativity of the sum.

Types. The barycentric $\lambda$-calculus is simply typed with the usual type system built on $A$ and $A \Rightarrow B$ with the restriction that barycentric sums of atomic terms are possible only if the latter have the same type. Decomposing $A \Rightarrow B$ with exponential and linear map: $! A \multimap B$, the type system can be reformulated within linear logic:

$$
\begin{aligned}
& \frac{x \in \mathcal{V}}{\Gamma, x: A \vdash x: A} \quad \text { (var) } \\
& \frac{x: A \in \Gamma \vdash \mathbf{s}: B}{\Gamma \vdash \lambda x . \mathbf{s}: A \Rightarrow B} \\
& \frac{\Gamma \vdash \mathbf{s} \quad \Gamma \vdash \mathbf{T}}{\Gamma \vdash(\mathbf{s}) \mathbf{T}} \quad(\text { app }) \\
& \frac{\Gamma \vdash \mathbf{s}_{i}: A \sum_{i=1}^{n} a_{i}=1}{\Gamma \vdash \sum_{i=1}^{n} a_{i} \mathbf{s}_{i}: A} \quad(\text { sum }) \\
& \overline{\Gamma \vdash \mathrm{T}: \mathcal{B}} \quad \text { (true) } \quad \overline{\Gamma \vdash \mathrm{F}: \mathcal{B}} \quad \text { (false) } \\
& \frac{\Gamma \vdash \mathbf{s}: \mathcal{B} \quad \Gamma \vdash \mathbf{S}: \mathcal{B} \quad \Gamma \vdash \mathbf{R}: \mathcal{B}}{\Gamma \vdash \text { if } \mathbf{s} \text { then } \mathbf{S} \text { else } \mathbf{R}} \text { (cond) }
\end{aligned}
$$

Semantics We interpret the barycentric $\lambda$-calculus in LinFin through a standard translation of the $\lambda$-calculus in LL, extended to deal with the barycentric and boolean features as follows:

$$
\begin{aligned}
& \llbracket \sum_{i=1}^{n} a_{i} \mathbf{s}_{i} \rrbracket^{\Gamma}=\sum_{i=1}^{n} a_{i} \llbracket \mathbf{s}_{i} \rrbracket^{\Gamma} \quad \llbracket \mathrm{T} \rrbracket^{\Gamma}=(1,0), \quad \llbracket \mathrm{F} \rrbracket^{\Gamma}=(0,1), \\
& \llbracket \text { if } \mathbf{R} \text { then } \mathbf{S} \text { else } \mathbf{T} \rrbracket^{\Gamma}=\left(\llbracket \mathbf{R} \rrbracket_{t}^{\Gamma} \llbracket \mathbf{S} \rrbracket_{t}^{\Gamma}+\llbracket \mathbf{R} \rrbracket_{f}^{\Gamma} \llbracket \mathbf{T} \rrbracket_{t}^{\Gamma}, \llbracket \mathbf{R} \rrbracket_{t} \llbracket \mathbf{S} \rrbracket_{f}^{\Gamma}+\llbracket \mathbf{R} \rrbracket_{f}^{\Gamma} \llbracket \mathbf{T} \rrbracket_{f}^{\Gamma}\right) .
\end{aligned}
$$

Notice that since $\llbracket \mathcal{B} \rrbracket=\mathbb{k}^{2}$, the semantics of each term $\mathbf{S}$ of type $\mathcal{B}$ is given by its two components $\llbracket \mathbf{S} \rrbracket=\left(\llbracket \mathbf{S} \rrbracket_{t}, \llbracket \mathbf{S} \rrbracket_{f}\right)$. 
Theorem 23. Totality finiteness spaces constitute a denotational model of the barycentric $\lambda$-calculus.

Hence, the notion of totality we have defined is in line with the notion of realisability in Logic where a term $\lambda x . \mathbf{t}: A \Rightarrow B$ is realisable iff $\forall \mathbf{s}: A, \mathbf{t}[x \leftarrow \mathbf{s}]: B$.

\section{Towards completeness}

We focus attention on closed terms of type $\mathcal{B}^{n} \Rightarrow \mathcal{B}$. As we have seen in Ex. 22, terms of that type are pairs of polynomials $\mathbb{P}=\left(\mathbb{P}_{t}, \mathbb{P}_{f}\right) \in \mathbb{k}\left[X_{1}, \ldots, X_{2 n}\right]^{2}$ s.t. for all $\left(a_{i}\right) \in \mathbb{k}^{2 n}$ with $a_{2 i-1}+a_{2 i}=1, \mathbb{P}_{t}\left(a_{1}, \ldots, a_{2 n}\right)+\mathbb{P}_{f}\left(a_{1}, \ldots, a_{2 n}\right)=1$.

Theorem 24 (Completeness). Every total function of $\mathcal{T}\left(\mathcal{B}^{n} \Rightarrow \mathcal{B}\right)$ is the interpretation of a term of the boolean barycentric calculus.

More precisely, we prove that every pair of polynomials $\mathbb{P} \in \mathcal{T}\left\langle\otimes^{n} ! \mathcal{B} \multimap \mathcal{B}\right\rangle$ is boolean, i.e. there is a term $\mathbf{S}$ of the boolean calculus such that $\llbracket \mathbf{S} \rrbracket=\left(\mathbb{P}_{t}, \mathbb{P}_{f}\right)$.

Let us first introduce some notations and intermediate results.

$$
\begin{aligned}
& \neg \mathbf{S}=\text { if } \mathbf{S} \text { then } \mathrm{F} \text { else } \mathrm{T}, \quad \llbracket \neg \mathbf{S} \rrbracket=\left(\llbracket \mathbf{S} \rrbracket_{f}, \llbracket \mathbf{S} \rrbracket_{t}\right) \text {, } \\
& \mathbf{S}^{+}=\text {if } \mathbf{S} \text { then } \mathrm{T} \text { else } \mathrm{T}, \quad \llbracket \mathbf{S}^{+} \rrbracket=\left(\llbracket \mathbf{S} \rrbracket_{t}+\llbracket \mathbf{S} \rrbracket_{f}, 0\right) \text {, } \\
& \mathbf{S}^{-}=\text {if } \mathbf{S} \text { then } \mathrm{F} \text { else } \mathbf{F}, \quad \llbracket \mathbf{S}^{-} \rrbracket=\left(0, \llbracket \mathbf{S} \rrbracket_{t}+\llbracket \mathbf{S} \rrbracket_{f}\right) \text {, } \\
& \boldsymbol{\Pi}_{i}=\lambda \mathbf{x}_{1}, \ldots, \mathbf{x}_{n} \cdot \mathbf{x}_{i}, \quad \llbracket \mathbf{\Pi}_{i} \rrbracket=\left(X_{2 i-1}, X_{2 i}\right) .
\end{aligned}
$$

The following pairs of polynomials are boolean:

$$
\begin{gathered}
\left(X_{2 i}, X_{2 i-1}\right)=X_{2 i} \cdot(1,0)+X_{2 i-1} \cdot(0,1)=\llbracket \neg \Pi_{i} \rrbracket, \\
\left(X_{2 i-1}+X_{2 i}, 0\right)=X_{2 i} \cdot(1,0)+X_{2 i-1} \cdot(1,0)=\llbracket \Pi_{i}^{+} \rrbracket, \\
\left(1-X_{2 i}, X_{2 i}\right)=(1,0)+\left(X_{2 i-1}, X_{2 i}\right)-\left(X_{2 i-1}+X_{2 i}, 0\right)=\llbracket \mathrm{T}+\Pi_{i}-\Pi_{i}^{+} \rrbracket, \\
\left(1-X_{2 i-1}, X_{2 i-1}\right)=\llbracket \mathrm{T}+\neg \boldsymbol{\Pi}_{i}-\mathbf{\Pi}_{i}^{+} \rrbracket .
\end{gathered}
$$

We prove first a weak version of the completeness theorem where we assume that $\mathbb{P}_{t}+\mathbb{P}_{f}-1$ vanishes everywhere.

Lemma 25 (Affine pairs). For every polynomial $P \in \mathbb{k}\left[X_{1}, \ldots, X_{n}\right]$, the pair of polynomials $(1-P, P)$ is boolean.

Proof. We use an induction on the degree $d$ of $P$. If $d=0$, there exists $a \in \mathbb{k}$ such that $P=a$, hence $(1-P, P)=\llbracket a \mathrm{~T}+(1-a) \mathrm{F} \rrbracket$.

If $d>0$, let us first study the monomial case, i.e. $X^{\mu}=\prod X_{i}^{\mu_{i}}$ with, say, $\mu_{1} \geq 1$.

$$
\begin{aligned}
\left(1-X^{\mu}, X^{\mu}\right) & =\left(1-X_{1}\right) \cdot(1,0)+X_{1} \cdot\left(1-X_{1}^{\mu_{1}-1} \prod_{i \neq 1} X_{i}^{\mu_{i}}, X_{1}^{\mu_{1}-1} \prod_{i \neq 1} X_{i}^{\mu_{i}}\right) \\
& =\llbracket \text { if } \boldsymbol{\Xi}_{1} \text { then T else } \boldsymbol{\Xi}_{d-1} \rrbracket=\llbracket \boldsymbol{\Xi}_{\mu} \rrbracket .
\end{aligned}
$$

where the induction hypothesis ensures the existence of $\boldsymbol{\Xi}_{1}$ and $\boldsymbol{\Xi}_{d-1}$ respectively interpreted by $X_{1}^{\mu_{1}-1}$ and $\prod_{i \neq 1} X_{i}^{\mu_{i}}$. Finally, if $P=\sum a_{\mu} \prod X_{i}^{\mu_{i}}$, then

$$
\begin{aligned}
(1-P, P) & =\left(1-\sum a_{\mu}\right)(1,0)+\left(\sum a_{\mu}\right)\left(1-X^{\mu}, X^{\mu}\right) \\
& =\llbracket\left(1-\sum a_{\mu}\right) \mathrm{T}+\left(\sum a_{\mu}\right) \boldsymbol{\Xi}_{\mu} \rrbracket .
\end{aligned}
$$


The following algebraic lemma, allows us to reduce our problem to Affine pairs.

Lemma 26 (Spanning polynomials). Let $P \in \mathbb{k}\left[X_{1}, \ldots, X_{2 n}\right]$ where $\mathbb{k}$ is an infinite field. If $P$ vanishes on the common zeroes of $X_{2 i-1}+X_{2 i}-1$, then for every $i$ in $\{1, \ldots, n\}$ there is $Q_{i} \in \mathbb{k}\left[X_{1}, \ldots, X_{2 n}\right]$ such that $P=\sum_{i=1}^{n} Q_{i}\left(X_{2 i-1}+X_{2 i}-1\right)$.

Proof. Under the change of variable: $Y_{i}=X_{2 i-1}+X_{2 i}-1, \quad Y_{i+n}=X_{2 i}, \quad$ for $i \in\{1, \ldots, n\}$, we denote by $P_{Y}$ the polynomial $P$. Then for every $\left(y_{i}\right) \in \mathbb{k}^{n}$, $P_{Y}\left(0, \ldots, 0, y_{n+1}, \ldots, y_{2 n}\right)=0$. Since $\mathbb{k}_{k}\left[Y_{2}, \ldots, Y_{2 n}\right]$ is a ring, $\mathbb{k}_{k}\left[Y_{2}, \ldots, Y_{2 n}\right]\left[Y_{1}\right]$ is an euclidean ring. The euclidean division of $P_{Y}$ by $Y_{1}$ gives $P_{Y}=Q_{1} Y_{1}+R_{1}$ where $R_{1} \in \mathbb{k}_{\mathrm{k}}\left[Y_{2}, \ldots, Y_{2 n}\right]\left[Y_{1}\right]$ and $R_{1}^{\prime} \in \mathbb{k}_{\mathrm{k}}\left[Y_{2}, \ldots, Y_{2 n}\right]$. By iterating this process on $R_{i}$ for $i \in\{1 \ldots n-1\}$, we get $P_{Y}=\sum_{i=1}^{n} Q_{i} Y_{i}+R_{n}$ where $R_{i} \in \mathbb{k}\left[Y_{1}, \ldots, Y_{2 n}\right]$ and $R_{n} \in \mathbb{k}\left[Y_{n+1}, \ldots, Y_{2 n}\right]$. For all $\left(y_{i}\right) \in \mathbb{k}^{n}$, we have

$P_{Y}\left(0, \ldots, 0, y_{n+1}, \ldots, y_{2 n}\right)=R_{n}\left(y_{n+1}, \ldots, y_{2 n}\right)=0$. Since $\mathbb{k}$ is infinite, $R_{n}=0$ and $P_{Y}=\sum_{i=1}^{n} Q_{i} Y_{i}$. Change the variables, we get, $P=\sum_{i=1}^{n} Q_{i}\left(X_{2 i-1}+X_{2 i}-1\right)$.

Proof (Theorem 24). Let $\mathbb{P} \in \mathcal{T}\left\langle\otimes^{n} ! \mathcal{B} \multimap \mathcal{B}\right\rangle$. Thanks to Ex. 22, we know that $\mathbb{P}_{t}+$ $\mathbb{P}_{f}-1$ vanishes on every zero of $\left\{X_{2 i-1}+X_{2 i}-1 \mid 1 \leq i \leq n\right\}$. Then, we can apply Lem. 26: $\mathbb{P}_{t}+\mathbb{P}_{f}-1=\sum_{i=1}^{n} Q_{i}\left(X_{2 i-1}+X_{2 i}-1\right)$ with $Q_{i} \in \mathbb{k}\left[X_{1}, \ldots, X_{2 n}\right]$. Thus, $\left(\mathbb{P}_{t}, \mathbb{P}_{f}\right)=\sum_{i=1}^{n}\left[\left(1-Q_{i}\right) \cdot(1,0)+Q_{i} \cdot\left(X_{2 i-1}+X_{2 i}, 0\right)\right]+\left(1-\mathbb{P}_{f}, \mathbb{P}_{f}\right)-n(1,0)$. By Lem. 25, there are boolean terms $\mathbf{S}_{i}$ and $\mathbf{T}$ such that $\left(1-Q_{i}, Q_{i}\right)=\llbracket \mathbf{S}_{i} \rrbracket$ and $\left(1-\mathbb{P}_{f}, \mathbb{P}_{f}\right)=\llbracket \mathbf{T} \rrbracket$. We have seen in Eq. (8) that $\left(X_{2 i-1}+X_{2 i}, 0\right)=\llbracket \boldsymbol{\Pi}_{i}^{+} \rrbracket$. Finally, we have found a term whose semantics is

$$
\mathbb{P}=\llbracket \sum_{i=1}^{n}\left(\text { if } \mathbf{S}_{i} \text { then } \mathbf{T} \text { else } \mathbf{\Pi}_{i}^{+}\right)+\mathbf{T}-n \mathrm{~T} \rrbracket .
$$

Example 27 (Gustave and Por functions). Several pairs of polynomials can interpret the functions $\mathrm{POr} \in \mathcal{T}(! \mathcal{B} \otimes ! \mathcal{B}) \multimap \mathcal{B}$ and Gus $\in \mathcal{T}(! \mathcal{B} \otimes ! \mathcal{B} \otimes ! \mathcal{B}) \multimap \mathcal{B}$ satisfying:

$$
\begin{array}{ll}
\operatorname{POr}(\mathrm{T}, 0)=\mathrm{T} & \operatorname{Gus}(\mathrm{T}, 0,0)=\mathrm{T} \\
\operatorname{POr}(0, \mathrm{~T})=\mathrm{T} & \operatorname{Gus}(0, \mathrm{~T}, 0)=\mathrm{T} \\
\mathrm{POr}(\mathrm{F}, \mathrm{F})=\mathrm{F} & \operatorname{Gus}(0,0, \mathrm{~T})=\mathrm{T} \\
& \operatorname{Gus}(\mathrm{F}, \mathrm{F}, \mathrm{F})=\mathrm{F}
\end{array}
$$

The pairs of polynomials with the smallest degree are respectively:

$$
\begin{aligned}
\text { POr }: & \mathcal{B} \times \mathcal{B} \Rightarrow \mathcal{B} \\
& (x, y) \mapsto\left(x_{t}+y_{f}-x_{t} y_{t}, x_{f} y_{f}\right) \\
\text { Gus : } & \mathcal{B} \times \mathcal{B} \times \mathcal{B} \Rightarrow \mathcal{B} \\
& (x, y, z) \mapsto\left(x_{t} y_{f}+y_{t} z_{f}+z_{t} x_{f}, x_{t} y_{t} z_{t}+x_{f} y_{f} z_{f}\right)
\end{aligned}
$$

\section{Conclusion}

The first two sections of this article emphasise the algebraic and topological description of the model of finiteness spaces. It is important for three reasons.

First, the definition of linear finiteness spaces is so web oriented that the corresponding 
category is not obviously closed by certain operations such as quotients or even subspaces. The purpose of a more algebraic approach is to get rid of bases. Our description of reflexivity is a first step in this direction.

Second, our study has unveiled an algebraic approach to totality where totality candidates admit a simple algebraic and topological characterisation; such a characterisation was not available in coherence spaces. Moreover, although we needed to use linear logic to describe algebraic totality, we get a notion which coincides with the standard intuitionist hierarchy. Notice that the non-stable POr function is total and hence definable. Consequently, stability and totality seems to be unrelated in this setting.

Finally, the partial completeness result is proved using an algebraic method. This gives a new insight into the analogy between linear algebra and linear logic. We hope to get completeness at other types or fragments of linear logic. But the result can already be compared with hypercoherences in which completeness holds at first order thanks to sequentiality $[4,16]$.

Acknowledgements I want to thank Pierre Hyvernat who was interested in the completeness part of this work. He gave another proof of the result stated here with an elegant combinatorial approach and found the total description of the Por function.

\section{References}

1. M. Barr. *-Autonomous Categories and Linear Logic. Mathematical Structures in Computer Science, 1(2):159-178, 1991.

2. RF Blute and P.J. Scott. Linear Lauchli semantics. Annals of Pure and Applied Logic, 77(2):101-142, 1996.

3. PH Chu. Autonomous categories, chapter Constructing*-autonomous categories. Lecture Notes in Mathematics, 11, 1979.

4. L. Colson and T. Ehrhard. On strong stability and higher-order sequentiality. In Logic in Computer Science, 1994. LICS'94. Proceedings., Symposium on, pages 103-108, 1994.

5. Thomas Ehrhard. On finiteness spaces and extensional presheaves over the lawvere theory of polynomials. Journal of Pure and Applied Algebra, to appear.

6. Thomas Ehrhard. Finiteness spaces. Mathematical. Structures in Comp. Sci., 15(4):615-646, 2005.

7. H.R. Fischer and H. Gross. Tensorprodukte linearer topologien. Math. Annalen, 160, 1965.

8. Jean-Yves Girard. The system F of variable types, fifteen years later. Theoretical Computer Science, 45:159-192, 1986.

9. Jean-Yves Girard. Linear logic. Theor. Comput. Sci., 50:1-102, 1987.

10. A Grothendieck. Produits tensoriels topologiques et espaces nucléaires, volume 16. American Mathematical Society, 1955.

11. Martin Hyland and Andrea Schalk. Glueing and orthogonality for models of linear logic. Theoretical Computer Science, 294(1-2):183-231, 2003.

12. Gottfried Köthe. Topological Vector Spaces I. Springer-Verlag, 1979.

13. Y. Lafont and T. Streicher. Games semantics for linear logic. In Logic in Computer Science, 1991. LICS'91., Proceedings of Sixth Annual IEEE Symposium on, pages 43-50, 1991.

14. Solomon Lefschetz et al. Algebraic topology. American Mathematical Society, 1942.

15. Ralph Loader. Linear logic, totality and full completeness. Logic in Computer Science, 1994. LICS'94. Proceedings., Symposium on, pages 292-298, 1994.

16. J. Longley. The sequentially realizable functionals. Annals of Pure and Applied Logic, 117(1-3):1-93, 2002. 
17. Lionel Vaux. On linear combinations of $\lambda$-terms. In Franz Baader, editor, RTA, volume 4533 of Lecture Notes in Computer Science, pages 374-388. Springer, 2007. 


\section{A Finiteness spaces}

Proposition 11. Let $K$ be a subspace of $\mathbb{k}\langle A\rangle$. There is an equivalence between
1. $K$ is linearly bounded,
2. $|K|=\cup\{|x| \mid x \in K\}$ is finitary,

3. the closure of $K$ is linearly compact.

Proof. Let $K$ be linearly bounded. Let $J^{\prime} \in \mathcal{F}(A)^{\perp}$. There is a finite dimensional subspace $K_{0}$ of $K$ such that $K=\left(K \cap V_{J^{\prime}}\right) \oplus K_{0}$. Since the dimension of $K_{0}$ is finite, $\left|K_{0}\right|$ is finitary. Besides, $|K| \cap J^{\prime}=\left|K_{0}\right| \cap J^{\prime}$ which is finite. We proved that $|K| \in \mathcal{F}(K)$.

Let $K$ be a subspace of $\mathbb{k}\langle A\rangle$ such that $|K|$ is finitary. The topology induced by $\mathbb{k}\langle A\rangle$ on its subspace $\mathbb{k}^{|K|}$ is the product topology. Indeed, it is generated by $\mathbb{k}^{|K|} \cap V_{J^{\prime}}=\left\{x \in \mathbb{k}^{|K|}|| x \mid \cap J^{\prime}=\emptyset\right\}$ with $J^{\prime} \in \mathcal{F}(A)^{\perp}$ and so $|K| \cap J^{\prime}$ is finite. By Tychonov Th. $8, \mathbb{k}^{|K|}$ is linearly compact. Since the closure of $K$ is a closed subspace of $\mathbb{k}^{|K|}$, it is also linearly compact (cf. [12, §10.9(1)]).

Let $K$ be a linearly compact subspace of $\mathbb{k}\langle A\rangle$. Let $J^{\prime} \in \mathcal{F}(A)^{\perp}$. Thanks to the incomplete basis theorem, there is $D$ such that $K=\left(K \cap V_{J^{\prime}}\right) \oplus D$. If we endow $D$ with the discrete topology and $K \cap V_{J^{\prime}}$ with the topology induced by $K$, then the projections on each subspace are continuous. Therefore, $D$ is linearly compact as the image of a linearly compact space by a continuous function [12, §10.9(2)]. Besides, $D$ has a finite dimension as every discrete linearly compact space.Thus $K$ is linearly bounded.

Proposition 13 (Separation). For every closed subspace $D$ of $\mathbb{k}\langle A\rangle$ and $x \notin D$, there is a continuous linear form $x^{\prime} \in \mathbb{k}\langle A\rangle^{\prime}$ such that $\left\langle x, x^{\prime}\right\rangle=1$ and $\forall d \in D,\left\langle d, x^{\prime}\right\rangle=0$.

Proof. Since $\cap V_{J^{\prime}}=\{0\}$, there is $J^{\prime} \in \mathcal{F}(A)^{\perp}$ such that $x \notin V_{J^{\prime}}$. Thanks to the incomplete basis theorem, we can define a linear form $x^{\prime}$ not necessarily continuous such that $\left\langle x^{\prime}, x\right\rangle=1$ and $\forall y \in D+V_{J^{\prime}},\left\langle x^{\prime}, y\right\rangle=0$. Since $V_{J^{\prime}} \subset \operatorname{ker} x^{\prime}, x^{\prime}$ is continuous.

Lemma 14. Let $F^{\prime} \subset \mathbb{k}\langle A\rangle^{\prime}$ finite. If $0 \notin$ aff $\left(F^{\prime}\right)$ then there exists $x \in E$ such that $\forall x^{\prime} \in F^{\prime},\left\langle x^{\prime}, x\right\rangle=1$.

Proof. Let $x_{1}^{\prime}, \ldots, x_{n}^{\prime} \in F^{\prime}$ be a maximal linearly independent collection. We first prove that $F^{\prime} \subseteq$ aff $\left(x_{1}^{\prime}, \ldots, x_{n}^{\prime}\right)$ : Let $x^{\prime} \in F^{\prime}$. Since $\left(x_{i}^{\prime}\right)$ is maximal, there exist $\lambda_{1}, \ldots, \lambda_{n}$ such that $x^{\prime}=\sum_{i=1}^{n} \lambda_{i} x_{i}^{\prime}$. Assume $0 \notin$ aff $\left(F^{\prime}\right)$, then the equation in $\mu_{i}$ : $0=\left(1-\sum_{i=1}^{n} \mu_{i}\right) x^{\prime}+\sum_{i=1}^{n} \mu_{i} x_{i}^{\prime}$ cannot have any solution. By replacing $x^{\prime}$ by $\sum_{i} \lambda_{i} x_{i}^{\prime}$ and since $\left(x_{i}\right)$ is independent, we get the system $\left\{\left(1-\sum_{i=1}^{n} \mu_{i}\right) \lambda_{j}+\mu_{j} \mid 1 \leq j \leq n\right\}$ which has no solution. Hence, its determinant $(-1)^{n-1}\left(1-\sum_{i=1}^{n} \lambda_{i}\right)$ is null and $\sum_{i=1}^{n} \lambda_{i}=1$. Hence $x^{\prime} \in$ aff $\left(x_{1}^{\prime}, \ldots, x_{n}^{\prime}\right)$. Since $x_{1}^{\prime}, \ldots, x_{n}^{\prime}$ are linearly independent, there exists $x \in E$ such that for any $1 \leq i \leq n,\left\langle x_{i}^{\prime}, x\right\rangle=1$, hence $\forall x^{\prime} \in F^{\prime} \subseteq \operatorname{aff}\left(x_{1}^{\prime}, \ldots, x_{n}^{\prime}\right),\left\langle x^{\prime}, x\right\rangle=1$.

Proposition 14 (Separation in the dual). Let $T^{\prime}$ be a closed affine subspace of $\mathbb{k}\langle A\rangle^{\prime}$ such that $0 \notin T^{\prime}$. There exists $x \in \mathbb{k}\langle A\rangle$ such that $\forall x^{\prime} \in T^{\prime},\left\langle x^{\prime}, x\right\rangle=1$. 
Proof. The closed subspace $T^{\prime}$ does not contain 0 , hence there exists a fundamental linear neighbourhood of 0 , that is ann $(K)$ where $K$ linearly compact in $\mathbb{k}\langle A\rangle$, such that $\operatorname{ann}(K) \cap T^{\prime}=\emptyset$. For any finite subspace $F^{\prime} \subseteq T^{\prime}$, let $T_{F^{\prime}}=\left\{x \in E \mid \forall x^{\prime} \in\right.$ $\left.F^{\prime},\left\langle x^{\prime}, x\right\rangle=1\right\}$. One has aff $\left(F^{\prime}\right) \subseteq T^{\prime}$ and hence aff $\left(F^{\prime}\right) \cap \operatorname{ann}(K)=\emptyset$. So $0 \notin$ aff $\left\{x_{\mid K}^{\prime} \mid x^{\prime} \in F^{\prime}\right\}$. Applying Lem. 14 in $K^{\prime}$, for every finite $F^{\prime} \subseteq T^{\prime}$, we get $x \in K$ such that $\forall x^{\prime} \in F^{\prime},\left\langle x^{\prime}, x\right\rangle=1$, so $x \in T_{F^{\prime}} \cap K$.

The collection $\left(T_{F^{\prime}}\right)$ where $F^{\prime}$ ranges over finite subsets of $T^{\prime}$ is a filter of closed affine subspaces of $\mathbb{k}_{k}\langle A\rangle$. All elements of this filter meet the linearly compact subspace $K \subseteq$ $\mathbb{k}\langle A\rangle$. Thus $K \cap \bigcap_{F^{\prime} \subseteq \text { fin }} T^{\prime} T_{F^{\prime}} \neq \emptyset$, so there is $x \in K$ such that $\forall x^{\prime} \in T^{\prime},\left\langle x^{\prime}, x\right\rangle=1$.

Proposition 15. (Equicontinuous spaces) Let A be a relational finiteness space. A subspace $B^{\prime}$ of $\mathbb{k}\langle A\rangle^{\prime}$ is linearly bounded if and only if there is $J^{\prime} \in \mathcal{F}(A)^{\perp}$ such that $B^{\prime} \subseteq \operatorname{ann}_{\mathbb{k}\langle A\rangle^{\prime}}\left(V_{J^{\prime}}\right)$.

Proof. First, $B^{\prime}$ is linearly bounded if and only if $\left|B^{\prime}\right| \in \mathcal{F}(A)$ (see Prop. 11). Second, $\exists J^{\prime} \in \mathcal{F}(A), B^{\prime} \subseteq \operatorname{ann}\left(V_{J^{\prime}}\right) \Longleftrightarrow \exists J^{\prime} \in \mathcal{F}(A),\left|B^{\prime}\right| \subseteq J^{\prime} \Longleftrightarrow\left|B^{\prime}\right| \in \mathcal{F}(A)$.

Proposition 16 (Reflexivity). The map $\iota: \mathbb{k}\langle A\rangle \rightarrow \mathbb{k}\langle A\rangle^{\prime \prime}$ defined below is a topological isomorphism.

$$
\forall x \in \mathbb{k}\langle A\rangle, \iota(x): x^{\prime} \in \mathbb{k}\langle A\rangle^{\prime} \mapsto\left\langle x^{\prime}, x\right\rangle=x^{\prime}(x) .
$$

Proof. If $x \neq 0$, then there is $J \in \mathcal{F}(A)^{\perp}$ such that $x \notin V_{J}$. By separation Prop. 13, there is $x^{\prime} \in \mathbb{k}\langle A\rangle^{\prime}$ such that $\left\langle x^{\prime}, x\right\rangle=1$, hence $\iota$ is injective. Let $x^{\prime \prime} \in \mathbb{k}\langle A\rangle^{\prime \prime}$ such that $x^{\prime \prime} \neq 0$. Let $x^{\prime} \in \mathbb{k}\langle A\rangle$ such that $\left\langle x^{\prime \prime}, x^{\prime}\right\rangle=1$. Thanks to separation in the dual Prop. 14, there is $x \in \mathbb{k}\langle A\rangle$ such that $\left\langle x^{\prime}, x\right\rangle=1$ and for all $y^{\prime} \in \operatorname{ker}_{\mathbb{k}}\langle A\rangle^{\prime}\left(x^{\prime \prime}\right)$, $\left\langle y^{\prime}, x\right\rangle=0$. Hence $\iota(x)$ and $x^{\prime \prime}$ coincide on both $x^{\prime}$ and $\operatorname{ker}\left(x^{\prime \prime}\right)$.

If $J^{\prime} \in \mathcal{F}(A)^{\perp}$, then the support $\left|\operatorname{ann}_{\mathbb{k}\langle A\rangle^{\prime}}\left(V_{J^{\prime}}\right)\right|=J^{\prime}$ is in $\mathcal{F}(A)^{\perp}$. By Prop. 11, $K_{J^{\prime}}=\operatorname{ann}_{\mathbb{k}\langle A\rangle^{\prime}}\left(V_{J^{\prime}}\right)$ is linearly compact in $\mathbb{k}\langle A\rangle^{\prime}$ and $\operatorname{ann}_{\mathbb{k}}\langle A\rangle^{\prime \prime}\left(K_{J^{\prime}}\right)$ is a linear neighbourhood of 0 in $\mathbb{k}\langle A\rangle^{\prime \prime}$. Conversely, let $K^{\prime}$ be a linearly compact subspace of $\mathbb{k}\langle A\rangle^{\prime}$. By Propositions 15 and $11, \operatorname{ker}_{\mathbb{k}\langle A\rangle}\left(K^{\prime}\right)$ is open in $\mathbb{k}\langle A\rangle$.

We next show that the Topological dual construction of linear finiteness spaces coincides with the orthogonal construction of relational finiteness spaces.

Proposition 17. The linear finiteness space $\mathbb{k}\langle A\rangle^{\prime}$ endowed with the linearly compact open topology is isomorphic to the linear finiteness spaces $\mathbb{k}\left\langle A^{\perp}\right\rangle$.

Proof. Let $x^{\prime} \in \mathbb{k}^{|A|}$, then $x^{\prime} \in \mathbb{k}\langle A\rangle^{*}$ where $\left\langle x^{\prime}, x\right\rangle=\sum_{a \in|A|} x_{a}^{\prime} x_{a}$. We have the following equivalence: $x^{\prime}$ is continuous if and only if there is $J^{\prime} \in \mathcal{F}(A)^{\perp}$ such that $\forall x \in V_{J^{\prime}},\left\langle x^{\prime}, x\right\rangle=\sum_{a \in|A|} x_{a}^{\prime} x_{a}=0$, that is $|x|^{\prime} \subset J^{\prime}$. Consequently, we have that $x^{\prime} \in \mathbb{k}\langle A\rangle^{\prime}$ if and only if $x^{\prime} \in \mathbb{k}\left\langle A^{\perp}\right\rangle$.

Let $J \in \mathcal{F}(A)$. The subspace $\mathbb{k}^{J}$ of $\mathbb{k}\langle A\rangle$ is linearly compact thanks to Prop. 11 . Hence ann $\left(\mathbb{k}^{J}\right)=\left\{\left.x^{\prime}|| x^{\prime}\left|\in \mathcal{F}(A)^{\perp},\right| x\right|^{\prime} \cap J=\emptyset\right\}$ is open in $\mathbb{k}\langle A\rangle^{\prime}$. Conversely, if $K \subseteq \mathbb{k}\langle A\rangle$ is linearly compact, then $|K|$ is finitary (Prop. 11) and $V_{|K|}$ is a fundamental linear neighbourhood of 0 . Moreover, $V_{|K|} \subseteq$ ann $(K)$ hence ann $(K)$ is open in $\mathbb{k}\langle A\rangle^{\prime}$. We proved that the two topologies coincide. 


\section{B Interpretation of proofs in LinFin.}

Hypocontinuity can be generalised to multilinear functions:

Definition 18. Let $\left(A_{i}\right)_{i<n}$ be a finite collection of relational finiteness spaces. An $n$ linear form $\phi: \times_{i} \mathbb{k}\left\langle A_{i}\right\rangle \rightarrow \mathbb{k}$ is hypocontinuous if for any $\left(K_{i}\right)$ collection of linearly compact subspaces of $\mathbb{k}\left\langle A_{i}\right\rangle$ s (respectively), for any $i_{0}$ there exists a fundamental linear neighbourhood $U_{i_{0}}$ such that $\phi\left(\times A_{i}\right)=0$ where $A_{i}=K_{i}$ if $i \neq i_{0}$ and $A_{i_{0}}=U_{i_{0}}$.

Any proof of the sequent $\vdash \Gamma$ of formula of MELL+MIX+SUM is interpreted as a continuous linear form on $\llbracket \Gamma \rrbracket$. If $\Gamma=A_{1}, \ldots, A_{n}$ then a proof of the sequent can be expressed as a hypocontinuous $n$-linear on $\times_{i} \llbracket A_{i} \rrbracket^{\prime}$. Finally, if $\Gamma=\Gamma_{1}, A$, then a proof of this sequent can be equivalently described as a continuous linear function from $\llbracket \Gamma_{1} \rrbracket$ to $\llbracket A \rrbracket$. We freely use these different presentations in to describe proofs.

The interpretation of proofs of MELL+MIX+SUM are described on Fig. 3- 4 and is similar with the presentation of [13] except for exponentials.

\begin{tabular}{|c|c|}
\hline A proof $\pi$ & Its interpretation \\
\hline$\overline{\vdash A, A^{\perp}} \mathrm{AX}$ & $\begin{aligned} \llbracket \pi \rrbracket: \llbracket A \rrbracket^{\prime} \times \llbracket A \rrbracket & \rightarrow \mathbb{k} \\
x^{\prime}, \quad x & \mapsto\left\langle x^{\prime}, x\right\rangle\end{aligned}$ \\
\hline 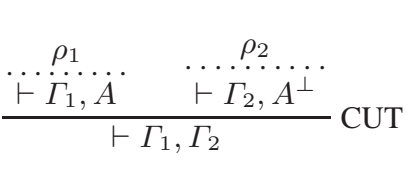 & $\begin{aligned} \llbracket \rho_{1} \rrbracket \in \llbracket \Gamma_{1}^{\perp} \multimap A \rrbracket & \llbracket \rho_{2} \rrbracket \in \llbracket \Gamma_{2}^{\perp} \multimap A^{\perp} \rrbracket \\
\llbracket \pi \rrbracket: \llbracket \Gamma_{1} \rrbracket^{\prime} \times \llbracket \Gamma_{2} \rrbracket^{\prime} & \rightarrow \mathbb{k} \\
\gamma_{1}^{\prime}, \quad \gamma_{2}^{\prime} & \mapsto\left\langle\rho_{2}\left(\gamma_{2}^{\prime}\right), \rho_{1}\left(\gamma_{1}^{\prime}\right)\right\rangle\end{aligned}$ \\
\hline$\frac{\stackrel{\rho_{i}}{\vdash} \ddot{\Gamma}}{\vdash \Gamma} \sum_{i=1}^{n} a_{i}=1$ & $\begin{aligned} \llbracket \rho_{i} \rrbracket \in \llbracket \Gamma \rrbracket^{\prime} & \sum_{i=1}^{n} a_{i}=1 \\
\llbracket \pi \rrbracket: \llbracket \Gamma \rrbracket & \rightarrow \mathbb{k}_{k} \\
\gamma & \mapsto \sum_{i=1}^{n} a_{i}\left\langle\llbracket \rho_{i} \rrbracket, \gamma\right\rangle\end{aligned}$ \\
\hline$\frac{}{\vdash \perp} \operatorname{MIX}_{0}$ & $\begin{aligned} \llbracket \pi \rrbracket: & \mathbb{k} \\
& \rightarrow \mathbb{k} \\
& a \mapsto a\end{aligned}$ \\
\hline 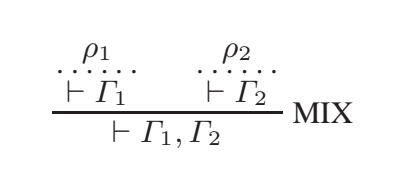 & $\begin{aligned} \llbracket \rho_{1} \rrbracket \in \llbracket \Gamma_{1} \rrbracket^{\prime} & \llbracket \rho_{2} \rrbracket \in \llbracket \Gamma_{2} \rrbracket^{\prime} \\
\llbracket \pi \rrbracket: \llbracket \Gamma_{1} \rrbracket^{\prime} \times \llbracket \Gamma_{2} \rrbracket^{\prime} & \rightarrow \mathbb{k} \\
\gamma_{1}^{\prime} \quad, \quad \gamma_{2}^{\prime} & \mapsto \llbracket \rho_{1} \rrbracket\left(\gamma_{1}^{\prime}\right) \llbracket \rho_{2} \rrbracket\left(\gamma_{2}^{\prime}\right)\end{aligned}$ \\
\hline$\frac{\stackrel{\rho}{\Gamma}}{\vdash \Gamma, \perp} \perp$ & $\begin{aligned} & \llbracket \rho \rrbracket \in \llbracket I \rrbracket^{\prime} \\
& \llbracket \pi \rrbracket: \llbracket \Gamma \rrbracket^{\prime} \times \mathbb{k} \rightarrow \mathbb{k} \\
& \gamma \quad, \quad a \mapsto \llbracket \rho \rrbracket\left(\gamma^{\prime}\right)\end{aligned}$ \\
\hline
\end{tabular}

Fig. 3: Interpretation of proofs of MELL+MIX+SUM in LinFin 


\begin{tabular}{|c|c|}
\hline$\frac{\rho}{\vdash \Gamma, \dddot{A}, \ddot{B}}$ & 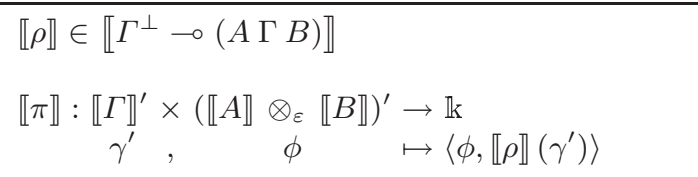 \\
\hline$\overline{\vdash 1}^{1}$ & $\begin{aligned} \llbracket \pi \rrbracket: & \mathbb{k} \\
a & \rightarrow \mathbb{k} \\
& \mapsto a\end{aligned}$ \\
\hline 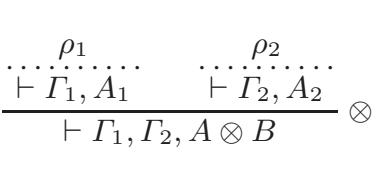 & $\begin{array}{l}\llbracket \rho_{1} \rrbracket \in \llbracket \Gamma_{1}^{\perp} \multimap A_{1} \rrbracket \quad \\
\llbracket \rho_{2} \rrbracket \in \llbracket \Gamma_{2}^{\perp} \multimap A_{2} \rrbracket \\
\llbracket \pi \rrbracket: \llbracket \Gamma_{1} \rrbracket^{\prime} \times \llbracket \Gamma_{2} \rrbracket^{\prime} \times\left(\llbracket A \rrbracket^{\prime} \otimes_{\varepsilon} \llbracket B \rrbracket\right) \rightarrow \mathbb{k} \\
\gamma_{1}^{\prime}, \quad \gamma_{2}^{\prime} \quad, \quad \phi \mapsto \phi\left(\llbracket \rho_{1} \rrbracket\left(\gamma_{1}^{\prime}\right), \llbracket \rho_{2} \rrbracket\left(\gamma_{2}^{\prime}\right)\right)\end{array}$ \\
\hline$\overline{\vdash \Gamma, \top} \top$ & $\begin{aligned} \llbracket \pi \rrbracket: \llbracket \Gamma \rrbracket^{\prime} \times\{0\} & \rightarrow \mathbb{k} \\
\gamma^{\prime} \quad, \quad 0 & \mapsto 0\end{aligned}$ \\
\hline 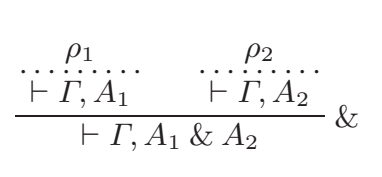 & $\begin{array}{l}\llbracket \rho_{1} \rrbracket \in \llbracket \Gamma^{\perp} \multimap A_{1} \rrbracket \quad \llbracket \rho_{2} \rrbracket \in \llbracket \Gamma^{\perp} \multimap A_{2} \rrbracket \\
\llbracket \pi \rrbracket: \\
\llbracket \Gamma \rrbracket^{\prime} \times\left(\llbracket A_{1} \rrbracket^{\prime} \oplus \llbracket A_{2} \rrbracket^{\prime}\right) \rightarrow \mathbb{k} \\
\gamma^{\prime}, x_{1}^{\prime}+x_{2}^{\prime} \mapsto\left\langle x_{1}^{\prime}, \llbracket \rho_{1} \rrbracket\left(\gamma^{\prime}\right)\right\rangle+\left\langle x_{2}^{\prime}, \llbracket \rho_{2} \rrbracket\left(\gamma^{\prime}\right)\right\rangle\end{array}$ \\
\hline$\frac{\stackrel{\rho}{\vdash \stackrel{\rho}{r} \ddot{A}_{1}}}{\vdash \Gamma, A_{1} \oplus A_{2}} \oplus_{g}$ & $\begin{array}{l}\llbracket \rho \rrbracket \in \llbracket \Gamma^{\perp} \multimap A_{1} \rrbracket \\
\llbracket \pi \rrbracket: \llbracket \Gamma \rrbracket^{\prime} \times\left(\llbracket A_{1} \rrbracket^{\prime} \times \llbracket A_{2} \rrbracket^{\prime}\right) \\
\gamma \quad, \quad\left(x_{1}^{\prime}, x_{2}^{\prime}\right) \quad \mapsto\left\langle x_{1}^{\prime}, \llbracket \rho \rrbracket(\gamma)\right\rangle\end{array}$ \\
\hline$\frac{\stackrel{\rho}{\vdash} \ddot{\Gamma}}{\vdash \Gamma, ? A^{\perp}}$ Weak & $\begin{array}{l}\llbracket \rho \rrbracket \in \llbracket \Gamma \rrbracket^{\prime} \\
\llbracket \pi \rrbracket: \llbracket \Gamma \rrbracket \times \llbracket ! A \rrbracket \\
\gamma \quad \rightarrow \mathbb{k} \\
\gamma \quad, \quad d \mapsto \llbracket \rrbracket(\gamma) \mathbb{1}_{d=e_{[}}\end{array}$ \\
\hline$\frac{\stackrel{\rho}{\vdash \Gamma, ? A^{\perp}, ? A^{\perp}}}{\vdash \Gamma, ? A^{\perp}}$ contr & $\begin{aligned} & \llbracket \rho \rrbracket \in \llbracket \Gamma \rrbracket \otimes_{\varepsilon} \llbracket ! A \rrbracket \otimes_{\varepsilon} \llbracket ! A \rrbracket \\
& \llbracket \pi \rrbracket: \llbracket \Gamma \rrbracket^{\prime} \times \llbracket ! A \rrbracket \rightarrow \mathbb{k} \\
& \gamma^{\prime}, \quad d \mapsto \sum_{d_{1}, d_{2}} \llbracket \rho \rrbracket\left(\gamma^{\prime}, d_{1}, d_{2}\right) \mathbb{1}_{d=d_{1}} \tilde{\otimes} d_{2}\end{aligned}$ \\
\hline$\frac{\rho}{\vdash \Gamma, A^{\perp}} \frac{\rho}{\vdash \Gamma, ? A^{\perp}}$ Der & $\begin{array}{l}\llbracket \rho \rrbracket \in \llbracket I \rrbracket \otimes_{\varepsilon} \llbracket A \rrbracket \\
\llbracket \pi \rrbracket: \llbracket I \rrbracket^{\prime} \times \llbracket ! A \rrbracket \rightarrow \mathbb{k} \\
\gamma^{\prime} \quad, \quad X \quad \mapsto \sum_{\# \mu=1} \llbracket \rho \rrbracket\left(\gamma^{\prime}, X_{\mu}\right)\end{array}$ \\
\hline$\frac{\vdash ? A_{1}^{\perp}, \ldots, ? A_{n}^{\perp}, B}{\vdash ? A_{1}^{\perp}, \ldots, ? A_{n}^{\perp}, ! B}$ Prom & $\begin{aligned} \llbracket \rho \rrbracket \in & \mathrm{Pol}^{n}\left(\llbracket A_{1} \rrbracket \times \cdots \times \llbracket A_{n} \rrbracket ; \llbracket A \rrbracket\right) \\
\llbracket \pi \rrbracket: & \mathrm{Pol}^{n}\left(\llbracket A_{1} \rrbracket \times \cdots \times \llbracket A_{n} \rrbracket ; \llbracket ! A \rrbracket\right) \\
& x_{1}, \ldots, x_{n} \mapsto\left(\llbracket \rho \rrbracket\left(x_{1}, \ldots, x_{n}\right)\right) !\end{aligned}$ \\
\hline
\end{tabular}

Fig. 4: Interpretation of proofs of MELL+MIX+SUM in LinFin 


\section{Totality candidate characterisation.}

Corollary 19. Let $\mathcal{T}$ be a subset of $\mathbb{k}\langle A\rangle$. If $\mathcal{T}^{\bullet} \neq \emptyset$, then $\mathcal{T}^{\bullet \bullet}=\overline{\operatorname{aff}}(\mathcal{T})$.

Proof. Since $\mathcal{T} \subseteq \mathcal{T}^{\bullet \bullet}$ and $\mathcal{T}^{\bullet \bullet}$ is affine close, we have $\mathcal{T} \subseteq \overline{\text { aff }}(\mathcal{T}) \subseteq \mathcal{T}^{\bullet \bullet}$. Consequently, $\mathcal{T}^{\bullet} \subseteq[\overline{\operatorname{aff}}(\mathcal{T})]^{\bullet} \subseteq \mathcal{T}^{\bullet}$ and $[\overline{\operatorname{aff}}(\mathcal{T})]^{\bullet}=\mathcal{T}^{\bullet} \bullet^{\circ}$. Moreover, if $\mathcal{T} \bullet \neq \emptyset$ then there is $x^{\prime} \in \mathbb{k}\langle A\rangle^{\prime}$ such that for any $x \in \mathcal{T},\left\langle x^{\prime}, x\right\rangle=1$, and so for any $x \in \overline{\operatorname{aff}}(\mathcal{T}),\left\langle x^{\prime}, x\right\rangle=1$. We infer that $0 \notin \overline{\text { aff }}(\mathcal{T})$. Thanks to the characterisation Lem. 18, $[\overline{\operatorname{aff}}(\mathcal{T})]^{\bullet \bullet}=\overline{\operatorname{aff}}(\mathcal{T})$. Finally, $\overline{\operatorname{aff}}(\mathcal{T})=\mathcal{T} \bullet$.

Proposition 21.

$$
\begin{aligned}
\mathcal{T}(A \otimes B) & =\overline{\operatorname{aff}}(\mathcal{T}(A) \otimes \mathcal{T}(B)), \\
\mathcal{T}(A \multimap B) & =\{f \in \mathbb{k}\langle A\rangle \mid f(\mathcal{T}(A)) \in \mathcal{T}(B)\} .
\end{aligned}
$$

Proof. If $x^{\prime} \in \mathcal{T}(A)^{\bullet}$ and $y^{\prime} \in \mathcal{T}(B)^{\bullet}$, then $x^{\prime} \otimes y^{\prime}:(x, y) \rightarrow\left\langle x^{\prime}, x\right\rangle\left\langle y^{\prime}, y\right\rangle$ is in $[\mathcal{T}(A) \otimes \mathcal{T}(B)]^{\bullet}$. Thanks to Cor. 19, $\overline{\text { aff }}(\mathcal{T}(A) \otimes \mathcal{T}(B))=[\mathcal{T}(A) \otimes \mathcal{T}(B)]^{\bullet \bullet}$.

The second equation comes from the equivalences:

$f \in\left[\mathcal{T}(A) \otimes \mathcal{T}(B)^{\bullet}\right]^{\bullet} \Longleftrightarrow \forall x \in \mathcal{T}(A), \forall y^{\prime} \in \mathcal{T}(B)^{\bullet},\left\langle f(x), y^{\prime}\right\rangle=1$, $\Longleftrightarrow \forall x \in \mathcal{T}(B), f(x) \in \mathcal{T}(B)$.

Proposition 22.

$$
\mathcal{T}(A \oplus B)=\overline{\operatorname{aff}}\left(\mathcal{T}(A) \times \operatorname{ker}\left(\mathcal{T}(B)^{\bullet}\right) \cup \operatorname{ker}\left(\mathcal{T}(A)^{\bullet}\right) \times \mathcal{T}(B)\right)
$$

Proof. By construction,

$\mathcal{T}(A \oplus B)=\left\{(x, y) \mid \forall u^{\prime} \in \mathcal{T}(A)^{\bullet}, v^{\prime} \in \mathcal{T}(B)^{\bullet},\left\langle x, u^{\prime}\right\rangle+\left\langle y, v^{\prime}\right\rangle=1\right\}$.

So $\overline{\text { aff }}\left(\mathcal{T}(A) \times \operatorname{ker}\left(\mathcal{T}(B)^{\bullet}\right) \cup \operatorname{ker}\left(\mathcal{T}(A)^{\bullet}\right) \times \mathcal{T}(B)\right) \subseteq \mathcal{T}(A \oplus B)$. Reciprocally, let $z=(x, y) \in \mathcal{T}(A \oplus B), u_{0}^{\prime} \in \mathcal{T}(A)^{\bullet}$ and $v_{0}^{\prime} \in \mathcal{T}(B)^{\bullet}, D(A)=\operatorname{dir}\left(\mathcal{T}(A)^{\bullet}\right)$ and $D(B)=\operatorname{dir}\left(\mathcal{T}(B)^{\bullet}\right)$. Then $\mathcal{T}(A)^{\bullet}=u_{0}^{\prime}+D(A)$ and $\mathcal{T}(B)^{\bullet}=v_{0}^{\prime}+D(B)$. Therefore for all $d_{x}^{\prime} \in D(A),\left\langle d_{x}^{\prime}, x\right\rangle=0$; for all $d_{y}^{\prime} \in D(B),\left\langle d_{y}^{\prime}, y\right\rangle=0$ and $\left\langle u_{0}^{\prime}, x\right\rangle+\left\langle v_{0}^{\prime}, y\right\rangle=1$. If $\left\langle v_{0}^{\prime}, y\right\rangle=0$ then $y \in \operatorname{ker}(D(B))$ and $x \in \mathcal{T}(A)$, so $z=$ $(x, y) \in \mathcal{T}(A) \times \operatorname{ker}\left(\mathcal{T}(A)^{\bullet}\right)$ (respectively, if $\left\langle u_{0}^{\prime}, x\right\rangle=0$, then $z \in \operatorname{ker}\left(\mathcal{T}(B)^{\bullet}\right) \times$ $\mathcal{T}(B))$. If $\left\langle u_{0}^{\prime}, x\right\rangle \neq 0$ and $\left\langle v_{0}^{\prime}, y\right\rangle \neq 0$, then $z=\left\langle u_{0}^{\prime}, x\right\rangle\left(\frac{x}{\left\langle u_{0}^{\prime}, x\right\rangle}, 0\right)+\left\langle v_{0}^{\prime}, y\right\rangle\left(0, \frac{y}{\left\langle v_{0}^{\prime}, y\right\rangle}\right)$. So $z=(x, y) \in \overline{\operatorname{aff}}\left(\mathcal{T}(A) \times \operatorname{ker}\left(\mathcal{T}(B)^{\bullet}\right) \cup \operatorname{ker}\left(\mathcal{T}(A)^{\bullet}\right) \times \mathcal{T}(B)\right)$.

Proposition 23.

$$
\begin{aligned}
\mathcal{T}(! A) & =\overline{\operatorname{aff}}\left(x^{!} \mid x \in \mathcal{T}(A)\right), \\
\mathcal{T}(? A) & =\{F \in \widetilde{\operatorname{Pol}}(\mathbb{k}\langle A\rangle) \mid \forall x \in \mathcal{T}(A), F(x)=1\}, \\
\mathcal{T}(! A \multimap B) & =\{F \in \widetilde{\operatorname{Pol}}(\mathbb{k}\langle A\rangle, B) \mid \forall x \in \mathcal{T}(A), F(x) \in \mathcal{T}(B)\} .
\end{aligned}
$$

Proof. Let $1 \in \operatorname{Pol}(\mathbb{k}\langle A\rangle)$ be the constant function: $\forall x \in \mathbb{k}\langle A\rangle,\left\langle x^{!}, 1\right\rangle=1$. We have $1 \in\left\{x^{!} \mid x \in \mathcal{T}(A)\right\}^{\bullet}$. Therefore, the first equality comes from Cor. 19. Using (9) and (10), we get (12). The equality (11) comes from the linear logic equivalence: ?A $!\left(A^{\perp}\right) \multimap 1$. 